\title{
Modeling the arrangement of particles in natural swelling-clay porous media using three-dimensional packing of elliptic disks
}

\author{
Eric Ferrage,,${ }^{1, *}$ Fabien Hubert, ${ }^{1}$ Emmanuel Tertre,${ }^{1}$ Alfred Delville, ${ }^{2}$ Laurent J. Michot, ${ }^{3}$ and Pierre Levitz ${ }^{3}$ \\ ${ }^{1}$ Université de Poitiers, CNRS, UMR 7285, Equipe HydrASA Laboratoire, Institut de Chimie des Milieux et Matériaux de Poitiers, \\ F-86022 Poitiers, France \\ ${ }^{2}$ Université d'Orléans, CNRS, UMR 7374, Interfaces, Confinement, Matériaux et Nanostructures, F-45071 Orléans, France \\ ${ }^{3}$ Université Pierre et Marie Curie, CNRS, UMR 8234, Physicochimie des Electrolytes et Nanosystèmes Interfaciaux, F- 72522 Paris, France
}

(Received 15 January 2015; published 15 June 2015)

\begin{abstract}
Swelling clay minerals play a key role in the control of water and pollutant migration in natural media such as soils. Moreover, swelling clay particles' orientational properties in porous media have significant implications for the directional dependence of fluid transfer. Herein we investigate the ability to mimic the organization of particles in natural swelling-clay porous media using a three-dimensional sequential particle deposition procedure [D. Coelho, J.-F. Thovert, and P. M. Adler, Phys. Rev. E 55, 1959 (1997)]. The algorithm considered is first used to simulate disk packings. Porosities of disk packings fall onto a single master curve when plotted against the orientational scalar order parameter value. This relation is used to validate the algorithm used in comparison with existing ones. The ellipticity degree of the particles is shown to have a negligible effect on the packing porosity for ratios $\ell_{a} / \ell_{b}$ less than 1.5 , whereas a significant increase in porosity is obtained for higher values. The effect of the distribution of the geometrical parameters (size, aspect ratio, and ellipticity degree) of particles on the final packing properties is also investigated. Finally, the algorithm is used to simulate particle packings for three size fractions of natural swelling-clay mineral powders. Calculated data regarding the distribution of the geometrical parameters and orientation of particles in porous media are successfully compared with experimental data obtained for the same samples. The results indicate that the obtained virtual porous media can be considered representative of natural samples and can be used to extract properties difficult to obtain experimentally, such as the anisotropic features of pore and solid phases in a system.
\end{abstract}

DOI: 10.1103/PhysRevE.91.062210

PACS number(s): 81.05.Rm, 61.43.Gt

\section{INTRODUCTION}

Swelling clay minerals (i.e., smectite and vermiculite) are composed of an octahedral sheet (with $\mathrm{Al}, \mathrm{Mg}, \mathrm{Fe}$, etc.) sandwiched between two opposing tetrahedral sheets (with $\mathrm{Si}$, $\mathrm{Al}$, and $\mathrm{Fe}$ ). Isomorphic substitutions at either the tetrahedral or octahedral sites induce a permanent negative layer charge, which is compensated for by the presence of exchangeable cations in the interlamellar space. As a function of water activity, the sorption of water molecules in the interlamellar space induces a swelling of the crystal structure (see [1-3] and references therein). These minerals are one of the main components of soils, where they exert a key role in controlling water and pollutant migration in the environment. From a more applied perspective, swelling clay minerals are currently considered worldwide in the design of disposal facilities for hazardous wastes, where their role is to inhibit the migration of contaminants from wastes to the surrounding environment.

Numerous studies have focused on the dynamical properties of water molecules and cations in the interlamellar space of swelling clay minerals [4-18]. However, on larger spatial scales, the presence of strongly anisometric particle shapes and their mutual arrangement in porous media are most often associated with the development of anisotropy in interparticle pore networks [19-21]. Such preferential particle orientation has a considerable impact on the directional dependence of hydraulic permeability, which results in the preferential transfer of fluids and solutes [22]. Understanding

*Corresponding author: eric.ferrage@univ-poitiers.fr the anisotropic features of swelling clay porous media and their effect on the migration of water and solutes is thus of prime importance for many natural processes involving the transfer of water and solutes on the macroscopic scale. As an illustration, Churakov et al. $[23,24]$ recently demonstrated that the macroscopic diffusion coefficient of water in pure swelling clay-based materials could be correctly predicted based on (i) the elementary self-diffusion coefficient of water molecules in the interlamellar space commonly derived from molecular dynamics simulations and (ii) a representation of particle organization in swelling clay porous media.

The studies of Churakov et al. [23,24] stressed the need for establishing clear pictures of representative porous media for swelling clay minerals, which ideally should account for the actual distributions of particle size, shape, and orientation in a system. Among the different experimental techniques that allow for such three-dimensional (3D) characterization of pure swelling clay mineral samples, $\mathrm{x}$-ray tomography and/or focused-ion-beam nanotomography are the most widely considered [25-28]. These techniques, based on 3D image analysis, can in principle provide an in situ analysis of pore networks in natural or composite pure clay systems without any assumption regarding particle size and shape. However, a spatial resolution that allows for a wide range of porosities in pure swelling clay-based materials to be covered is still rather difficult to attain, especially for small pore sizes. Another approach for examining porous media applicable to clay minerals lies in the use of 3D particle packing simulation procedures. Such simulations provide ways of assessing the effects of different geometric parameters of porous media on water and solute migration in certain natural systems. 
TABLE I. Analysis of distribution of particle sizes and shapes in the different size fractions derived from Reinholdt et al. [34]. Experimental data are fitted using a log-normal distribution characterized by a mean value $\mu$, a median value $m=e^{\mu}$, and a standard deviation $\sigma$. The median values for the basal surface and circle diameter of an equivalent surface are reported in $\mu \mathrm{m}^{2}$ and $\mu \mathrm{m}$, respectively. The aspect ratio is the ratio between the thickness of the particle and the disk diameter of an equivalent surface. The ratio $\ell_{a} / \ell_{b}$ is the ratio between the semimajor and semiminor axes of the ellipse fitting the particle surface area.

\begin{tabular}{|c|c|c|c|c|c|c|c|c|}
\hline \multirow{2}{*}{$\begin{array}{l}\text { Size fraction } \\
(\mu \mathrm{m})\end{array}$} & \multicolumn{2}{|c|}{ Basal surface } & \multicolumn{2}{|c|}{ Disk diameter } & \multicolumn{2}{|c|}{ Aspect ratio } & \multicolumn{2}{|c|}{$\ell_{a} / \ell_{b}$} \\
\hline & $m$ & $\sigma$ & $m$ & $\sigma$ & $m$ & $\sigma$ & $m$ & $\sigma$ \\
\hline $0.1-0.2$ & 0.005 & 0.901 & 0.081 & 0.446 & 0.089 & 0.695 & 1.695 & 0.304 \\
\hline $1-2$ & 0.111 & 1.987 & 0.376 & 0.675 & 0.093 & 0.473 & 1.464 & 0.220 \\
\hline $10-20$ & 472.844 & 0.489 & 24.537 & 0.240 & 0.099 & 0.213 & 1.285 & 0.110 \\
\hline
\end{tabular}

Among the different procedures considered for generating 3D porous media, the sequential deposition algorithm proposed by Coelho et al. [29] has proven successful in (i) the simulation of random packing of particles with various shapes and (ii) the calculation of the transport properties through such media [29-31]. The present study focuses on developing an alternative algorithm (also based on the sequential deposition procedure) to obtain simulated 3D swelling clay porous media with the possibility of varying particle geometrical parameters and particle orientation. The development of such an algorithm is motivated by our recent experimental studies, which focused on model systems with tunable particle orientation that provide materials with varying degrees of anisotropy [32]. These synthetic systems were prepared from size-selected vermiculite particle fractions using a logarithmic-scale particle size progression $(0.1-0.2,1-2$, and $10-20 \mu \mathrm{m})$ representative of the wide size range encountered in soil environments [from $\sim 10 \mathrm{~nm}$ to $\sim 20 \mu \mathrm{m}$ (see [33] and references therein)]. Using a suite of microscopy techniques (i.e., atomic force, optical, and transmission electron microscopy), the geometrical properties of particles in the different size fractions were obtained [34]. Particle shapes were assimilated to elliptic disks to account for the angular edges of particles and their elongated and flat basal surfaces, a common property of clay mineral particles. The geometrical analysis carried out to date (Table I) has involved the measurement of the distribution of basal surfaces, aspect ratios, and ellipticity. Additional analyses of experimental rocking curves (RCs) on porous media built from filter cakes of these different size fractions revealed an almost purely isotropic system for the coarsest fraction, whereas the finest fractions displayed much more anisotropic features [32].

Section II of the present paper is devoted to the presentation of the sequential deposition algorithm that has been built along the lines originally proposed by Coelho et al. [29]. The main features of the algorithm will be described, with an emphasis on the procedure used for detecting particle contact and overlap. These features are particularly relevant in the case of natural particles with highly contrasting geometric dimensions (Table I). In Sec. III the algorithm is applied to the specific case of disklike particles. For disks with the same aspect ratio, we first analyze the effect of the degrees of freedom in particle motion during the settlement process on the final packing porosity. We then examine how the initial configuration of a particle, when introduced in the simulation box, affects the porosity of the final packings. The effect of particle aspect ratio on packing porosity is then investigated before the algorithm is validated by comparing the obtained results with existing data derived from both numerical and experimental procedures. In Sec. IV we extend the analysis of packing properties to the case of particles with elliptic disk shapes. Although previous studies have shown that the ellipticity degree of particles could affect the final porosities of bidimensional packings [35,36], a detailed study of this type of particle morphology with respect to $3 \mathrm{D}$ packings is lacking. In this section we also explore the influence of the size and shape distribution of particles on the obtained porosities. Finally, Sec. V is devoted to the simulation of particle organization in natural swelling-clay porous media. We first compare the surface and geometric properties of the obtained virtual packings with experimental data and then explore the properties of the obtained virtual porous media to extract useful stereological information regarding the pores and the solid phase.

\section{THREE-DIMENSIONAL SEQUENTIAL DEPOSITION ALGORITHM}

\section{A. General presentation of the algorithm}

As described by Coelho et al. [29], the simulation employed in this study is based on the sequential deposition of particles under a gravitational field in a square simulation box of width $w$ and with periodic conditions along the $x$ and $y$ axes ( $z$ axis pointing upward). The bottom of the simulation box (at $z=0$ ) is considered rigid. According to the sequential deposition algorithm schematized in Fig. 1, each particle is introduced at the top of the simulation box and settles either at the bottom of the box or onto an existing bed of particles with fixed positions. At each elementary step of the algorithm, the bottom surface of the particle and the edge surface are sequentially scanned, as detailed below, to detect the minimum difference in altitude $d_{\text {min }}$ between the particle and other particles from the existing bed. In the case in which $d_{\text {min }}$ is higher than the fixed contact distance along the $z$ axis $\left(d_{\text {cont }}\right)$, no contact point is detected and the particle encounters a vertical free fall with a random displacement amplitude ranging from 0 to $d_{\min }$. In the case in which $d_{\text {min }}<d_{\text {cont }}$, one or several contact points are detected and three types of movements are then randomly chosen to minimize the altitude of the particle according to the steepest descent method based on the position of the barycenter of the particle and that of the single or the several contact points. The particle can slide with a random distance value $\delta_{\text {slide }}$ ranging from 0 to the maximum distance amplitude $A_{\text {slide }}^{\max }$. The particle is also allowed to swivel over a random angular 


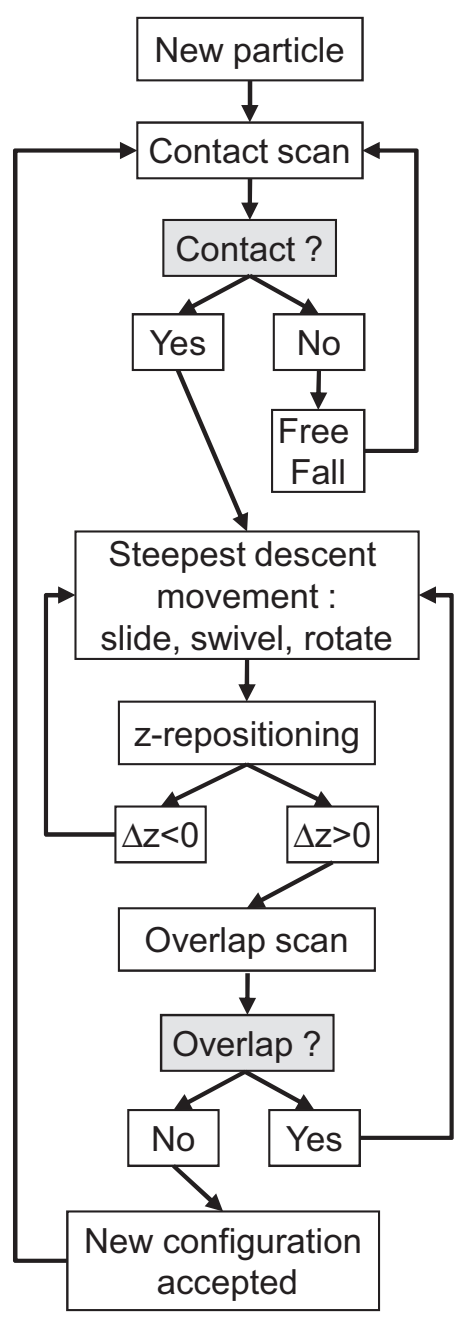

FIG. 1. Scheme of the sequential deposition algorithm used in the present study.

value $\omega_{\text {swiv }}$ (with $0<\omega_{\text {swiv }}<A_{\text {swiv }}^{\text {max }}$ ) around the contact point to provide the steepest descent of the barycenter altitude. Finally, for the specific case in which particles exhibit an elliptic surface shape, the particle is allowed to encounter an in-plane rotation of a random angular value $\omega_{\text {rot }}$ between zero and the maximum angular amplitude value $A_{\text {rot }}^{\max }$. As noted by Coelho et al. [29], in most cases, single contact points are detected. In the rare case in which two contact points are detected, the point around which motions are performed then corresponds to the intersection between the line connecting the two contact points and the altitude of the triangle formed by these two contact points and the particle barycenter. After movement, the particle is then vertically repositioned such that the new particle position satisfies the relation $d_{\text {min }}=d_{\text {cont }} / 2$. The difference in altitude $\delta z$ between the initial position of the particle barycenter and the new position after the movement attempt and the $z$ repositioning of the particle are evaluated. For $\delta z<0$, the particle altitude is increased by the movement. This movement attempt is thus rejected and the particle is returned to its initial configuration. In the case in which $\delta z>0$, a decrease in particle altitude is obtained and the final step of the algorithm involves the analysis of the potential overlap between particles (Fig. 1). This final step, detailed below, sequentially scans the potential overlap of particles. After the analysis, the movement attempt is rejected if overlap is detected, whereas in the opposite case, the new configuration is accepted and an additional particle movement is attempted.

Compared to the original algorithm proposed by Coelho et al. [29], the present algorithm is more straightforward because all amplitudes of motion are randomly generated. Moreover, the method does not use a conjugate gradient algorithm for the final steps of the settling process to limit the trapping of particles in nonoptimal positions. To help each particle find its final settled position, the present algorithm considers three cycles of 800 attempts for each particle and between each cycle the maximum amplitudes of translation and rotation (i.e., $A_{\text {slide }}^{\max }, A_{\text {swiv }}^{\max }$, and $A_{\text {rot }}^{\max }$ ) are divided by 2 . This modification allows each particle to limit the rejection of movement attempts when reaching the final settled position. Because the present study aims at simulating clay porous media composed of particles displaying a wide range of morphologies, most of the computational efforts in the present algorithm are dedicated to the detection of contacts and overlap between particles with highly contrasting shapes and sizes, as detailed in the following section.

\section{B. Geometrical description of particles and analysis of contacts and overlaps}

In its own reference frame, each particle is defined as a flat elliptic disk. The basal flat surface is described by a semimajor axis $\ell_{a}$ and a semiminor axis $\ell_{b}$, whereas the thickness of the particle is referred to as $h$. In the particle's reference frame, $\ell_{a}$, $\ell_{b}$, and $h$ are aligned along the $x, y$, and $z$ axes, respectively, whereas the barycenter is located at the origin of the reference frame. The particle aspect ratio $\mathcal{R}$ is calculated as the ratio between the thickness $h$ and the mean diameter $\tilde{d}$ of a circle with an equivalent basal surface area $\left(R=h / \tilde{d}=h / 2 \sqrt{\ell_{a} \ell_{b}}\right)$. Coordinate transformation between the frame of the particle and that of the simulation box is then ensured by providing each particle with a set of three Euler angles $\varphi, \theta$, and $\psi$ following the $Z X Z$ convention and the associated restrictions, i.e., $\varphi \in[0,2 \pi), \theta \in(0, \pi / 2), \in[0,2 \pi)$, as well as a position $\vec{r}$ of the particle barycenter in the simulation box. All 3D representations presented in this study were built using the Persistence of Vision ${ }^{\mathrm{TM}}$ Raytracer [37].

The scan of the minimum vertical distance $d_{\text {min }}$ between the settling particle and the other particles in the bed starts by applying, in the settling particle reference frame, a square grid to the bottom basal surface, as illustrated in Fig. 2(a). The vertices of each square represent the points of interest for the scan, whereas the precision of the contact detection scan relies on a predefined number of scan points $n_{s p}$ on the bottom basal surface, which ranges from 150 up to 500 in the present study. This configuration provides an elementary characteristic distance $\delta_{g 1}^{s}$ for the first scan grid of the settling particle, represented by the distance between two neighboring vertices of the same square. The same distance parameter $\delta_{g 1}^{s}$ is also used to define a number of scan points for the edge of the settling particle, as shown in Fig. 2(b). Thus, for a disk particle with an aspect ratio $\mathcal{R}=0.1$ and $n_{s p}=150$, approximately 300 scan points are defined in the particle reference frame. The $d_{\min }$ value is then analyzed sequentially [29,38]. For 
(a)
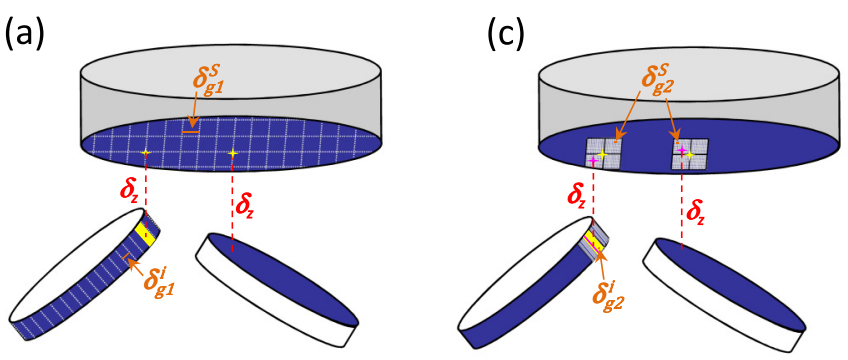

(b)
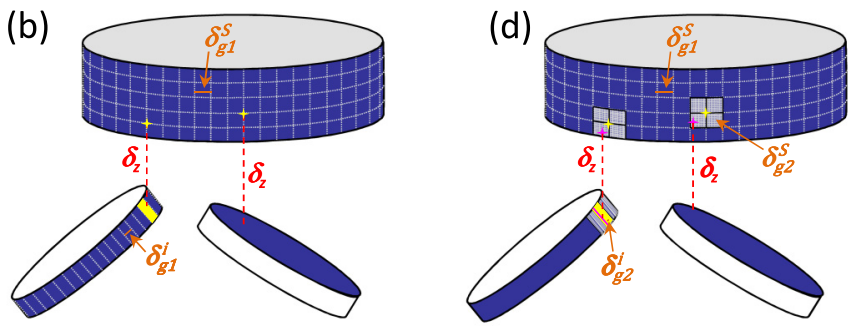

FIG. 2. (Color online) Sequential method used to detect contact between the (a) and (c) basal and (b) and (d) lateral surfaces of the settling particle and particles from the existing bed. See the text for details.

each scan point, the altitude $\delta_{z}$ is first calculated for basal surfaces and then for edge surfaces of particles already in place in the bed. For the calculation of $\delta_{z}$ distances with edge surfaces of particles in the bed, this latter type of surface is polygonized into a number of rectangular polygons calculated as the perimeter of the $i$ th particle from the bed divided by $\delta_{g 1}^{i}$ (i.e., the elementary characteristic distance of the $i$ th particle from the bed). After analysis, only scan points with altitude differences $\delta_{z} \leqslant \delta_{g 1}^{s}$ are retained for another round of calculation. In this second round, the elementary characteristic distance of the settling particle is defined as $\delta_{g 2}^{s}=\delta_{g 1}^{s} / 10$. Similarly, the characteristic distance for analyzing contact with the edge of a particle from the bed is also reduced such that $\delta_{g 2}^{i}=\delta_{g 1}^{i} / 10$. For each scan point of interest, the algorithm then identifies the position of the scan point in the range $\left(-\delta_{g 1}^{s} ;+\delta_{g 1}^{s}\right)$ and/or in the range $\left(-\delta_{g 1}^{i} ;+\delta_{g 1}^{i}\right)$ leading to the minimum value for $\delta_{z}$ for both the basal and the edge surfaces of the settling particle as illustrated in Figs. 2(c) and 2(d), respectively. At the end of the procedure, scan points with $\delta_{z} \leqslant \delta_{g 2}^{s}$ are again considered, whereas other scan points are excluded from this sequential analysis, which continues until the characteristic distance for the $n$th round of scanning $\left(\delta_{g n}^{s}\right)$ is less than the defined contact distance along the $z$ axis (i.e., $d_{\text {cont }}$ parameter). Once this condition is reached, the distance $\delta_{z}$ values are used to determine the number of contact points between the settling particle and other particles from the bed.

The analysis of the overlap between the settling particle and the others in the bed is also performed in a sequential fashion. The analysis again begins by defining a number of scan points (i.e., $n_{s p}$ as for the scan of contact points), applied for both the upper and bottom basal surfaces, to calculate an elementary characteristic distance $\delta_{g 1}^{i}$ in the coordinate reference frame of the $i$ th particle from the existing bed. The coordinates of these scan points are then transformed into the reference frame of the settling particle. In this settling particle reference frame, these scan points are excluded from further calculations if not contained in the envelope defined by $\left(\ell_{a}+\delta_{g 1}^{i}\right),\left(\ell_{b}+\delta_{g 1}^{i}\right)$, and $\left(h+2 \delta_{g 1}^{i}\right)$, whereas particle overlap is detected when the scan points are contained inside the settling particle. For other scan points, the analysis continues with $\delta_{g(n)}^{s}=$ $\delta_{g(n-1)}^{s} / 10$ until $\delta_{g(n)}^{i} \leqslant d_{\text {cont }}$. It should be emphasized that particle overlap remains possible and strongly depends on the value given to the $n_{s p}$ parameter. In the case where particle interpenetration is not detected during the first sequence of contact analysis, which represents most of the computational effort, there is a chance that such an effect will not be detected during the following sequences of analysis. This effect could be potentially aggravated in the case where a large size particle would settle on an extremely small particle. Such particle overlap in sequential deposition algorithms has been previously described in detail $[29,38]$. In the present case the consideration of an inverse procedure between the scan of contact points (based on an initial scan of the settling particle surfaces) and the scan of particle overlap (based on an initial scan of surfaces from the bed particles) is expected, however, to reduce potential interpenetration between particles.

\section{PACKING OF MONODISPERSE DISKS: COMPARISON WITH EXISTING ALGORITHMS}

In this section we explore the effects of different parameters of the sequential deposition algorithm on the final packing properties. First, the effect of maximum amplitude given to swivel and slide motions is assessed. Second, two series of calculations are used to investigate the effect of the initial orientation of a particle on the final porosity of the bed. All of these results for disks are then used for comparison with existing data to assess the validity of the present algorithm.

\section{A. Sensitivity to the maximum amplitude parameters for motions}

For the sensitivity tests, an aspect ratio $\mathcal{R}=0.1$ is considered for the disks to mimic the mean particle aspect ratio in our natural swelling-clay porous media (Table I). The width of the simulation box is set to $w=7.5$, whereas the characteristic contact distance along the $z$ axis $d_{\text {cont }}=$ $5 \times 10^{-4} \tilde{d}$. Each simulation involves a number of particles $N_{p}=2500$ generated with random values for angles $\varphi, \theta$, and $\psi$ and random initial $(x, y)$ positions. The influence of the maximum amplitude values for the swiveling ( $A_{\text {swiv }}^{\max }$ ) or sliding ( $\left.A_{\text {slide }}^{\max }\right)$ of the settling particle is investigated over the ranges of $5^{\circ} \leqslant A_{\text {swiv }}^{\max } \leqslant 80^{\circ}$ and $\tilde{d} / 60 \leqslant A_{\text {slide }}^{\max } \leqslant 7 \tilde{d}$, respectively.

The obtained packings are then compared based on their overall porosity $\varepsilon$ (i.e., void volume fraction). The $\varepsilon$ values of the porous media are obtained by first plotting a porosity profile along the $z$ axis of the simulation box. For each slice along the $z$ axis of thickness $d z$ and volume $w^{2} d z$, the local porosity is determined by summing the solid volumes of particles whose barycenter altitudes $r_{z}$ are found in the range $z \leqslant r_{z}<z+d z$. As illustrated in Fig. 3(a) for the packing calculated with $A_{\text {swiv }}^{\max }=40^{\circ}$ and $A_{\text {slide }}^{\max }=\tilde{d}$, the obtained porosity profile shown in Fig. 3(b) displays a decreasing tendency for $z<z_{\min }$. This trend is associated with the rigid bottom of the simulation box, leading to particles lying flat on the box surface, which 

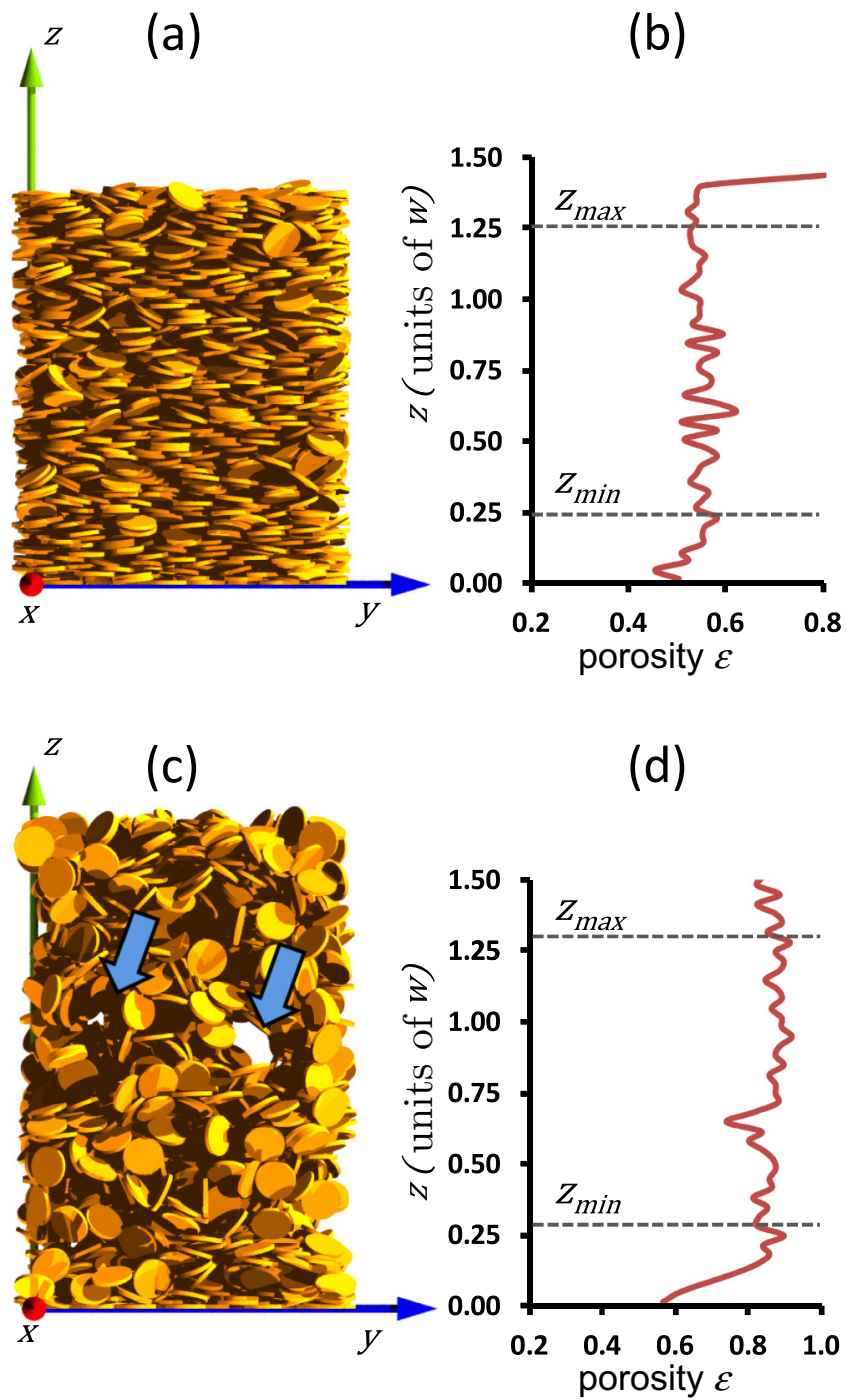

FIG. 3. (Color online) Packings obtained using (a) a high and (c) a low degree of freedom in particle motion. (b) and (d) are associated porosity profiles along the $z$-direction, respectively. The arrows in (c) highlight the presence of large pores in the packing. The overall porosity is calculated from the nearly linear part of the profile between $z_{\max }$ and $z_{\min }$.

in turn results in a slightly denser packing. On the top of the particle bed (i.e., for $z>z_{\max }$ ), the porosity values typically increase as a result of an incomplete filling of the simulation box. The $\varepsilon$ values calculated for all packings are thus extracted from the nearly linear part of the porosity for particles with barycenter altitude $r_{z}$ located in the range $z_{\min }<r_{z} \leqslant z_{\max }$. Although very simple, this procedure provides similar $\varepsilon$ values irrespective of the number of slices considered. However, an uncertainty of \pm 0.02 is attributed to all $\varepsilon$ values in the present study.

Figure 4(a) displays the effect of the maximum amplitude parameters on the obtained packing porosities. As a general trend, the obtained $\varepsilon$ values range between 0.84 and 0.51 and tend to decrease as $A_{\text {slide }}^{\text {max }}$ and $A_{\text {swiv }}^{\text {max }}$ increase. This relationship is fully consistent with the increase in the number of degrees of freedom in particle motion that allows for the solid to explore a larger number of positions and orientational configurations

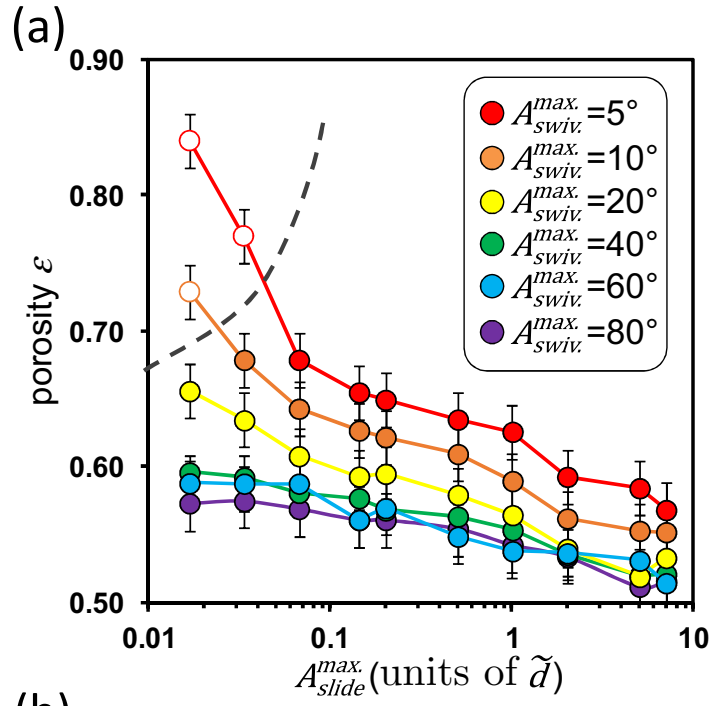

(b)



FIG. 4. (Color online) Effect of the algorithm parameters on the packing porosity $\varepsilon$ for disks with $R=0.1$. (a) Packings obtained with different values given to the maximum amplitude parameters for swivel $A_{\text {swiv }}^{\max }$ and slide $A_{\text {slide }}^{\max }$ motions. Open circles correspond to packings in which the presence of large pores, as shown in Fig. 3(c), is detected. (b) Packings obtained for different initial orientations $\theta_{\text {ini }}$ of the settling particles. Squares and circles correspond to data obtained with $\theta_{\text {ini }} \in\left(0, \theta_{\max }\right)$ and $\theta_{\text {ini }}=\theta_{\max }$, respectively. Closed and open symbols represent the packings obtained for low (i.e., $A_{\text {swiv }}^{\max }=10^{\circ}$ and $A_{\text {slide }}^{\max }=\tilde{d} / 7$ ) and high (i.e., $A_{\mathrm{swiv}}^{\max }=80^{\circ}$ and $A_{\text {slide }}^{\max }=5 \tilde{d}$ ) degrees of freedom in particle motion during the settlement process.

to minimize its barycenter altitude. The obtained packings are, however, weakly sensitive to the maximum amplitude for swivel motions when $A_{\text {swiv }}^{\text {max }} \geqslant 40^{\circ}$. For the same range of $A_{\text {swiv }}^{\max }$ values, a limited effect of the maximum amplitude for slide motions is also obtained for small and large $A_{\text {swiv }}^{\max }$ values (i.e., $A_{\text {slide }}^{\max }<0.1 \tilde{d}$ and $A_{\text {slide }}^{\max }>\tilde{d}$, respectively). It is worth noting that among the different porous media obtained, three systems exhibit $\varepsilon$ values greater than 0.7 , a situation that occurs when both $A_{\text {slide }}^{\max }$ and $A_{\text {swiv }}^{\text {max }}$ are close to their minimal values. Analysis of these three packings reveals peculiar features, as indicated by the arrows in Fig. 3(c) for the packing calculated with $A_{\text {swiv }}^{\max }=5^{\circ}$ and $A_{\text {slide }}^{\max }=\tilde{d} / 60$. Indeed, these three 
porous media display heterogeneous particle organization with individualization of large pores between particle aggregates. This behavior explains the large porosity values obtained and likely results from the very limited values considered for the maximum amplitude parameters. Indeed, with such a limited degree of freedom, the particles become rapidly trapped in local minima during the settling process, thus allowing for the formation of arched structures, leading to the individualization of large pores [38-40]. Such a heterogeneous organization cannot, however, be detected in the porosity profile along the $z$ axis reported for this packing in Fig. 3(d), which remains nearly linear throughout the sample.

\section{B. Sensitivity to the initial particle orientation}

In this second sensitivity test, we assess the effect of the initial orientational configuration of the particle when introduced in the simulation box, characterized by the initial particle angle $\theta_{\text {ini }}$ (angle between the normal unit vector of the particle surface and the $z$ axis of the simulation box), on the final packing porosity. For these calculations, random values are given to angles $\varphi$ and $\psi$ and two different ways of generating initial angles $\theta_{\text {ini }}$ are considered. In the first series of calculations, the angles $\theta_{\text {ini }}$ are randomly generated between 0 and a maximum angular value $\theta_{\max }$ defined as $\theta_{\max } \in(0, \pi / 2)$. This series mimics a preferential orientation of particles during their initial settlement. For the second series of simulations, a fixed $\theta_{\max }$ value, again defined as $\theta_{\max } \in(0, \pi / 2)$, is attributed to all particles. For each series, two sets of maximum amplitude parameters are considered to provide rather low (i.e., $A_{\text {swiv }}^{\max }=10^{\circ}$ and $A_{\text {slide }}^{\max }=\tilde{d} / 7$ ) and high (i.e., $A_{\text {swiv }}^{\max }=80^{\circ}$ and $A_{\text {slide }}^{\text {max }}=5 \tilde{d}$ ) degrees of freedom to particle motions during the settlement process. Other parameters considered for the deposition procedure, i.e., number of particles, particle aspect ratio, size of the simulation box, and contact distance, are identical to those used in the sensitivity tests discussed in Sec. III A.

As shown in Fig. 4(b), the value of $\theta_{\text {max }}$ significantly affects the packing porosity. Indeed, low porosity values are obtained when particles are generated with low $\theta_{\text {ini }}$ values, i.e., when their normal vector is superimposed with the $z$ axis of the simulation box. As a consequence, the obtained porosities are lower when the $\theta_{\max }$ values decrease. Moreover, for a given $\theta_{\max }$ value, lower porosities are logically obtained for $\theta_{\text {ini }} \in\left(0, \theta_{\max }\right)$ compared to the case in which $\theta_{\text {ini }}=\theta_{\text {max }}$. This difference between the two sets of $\theta_{\text {ini }}$ values generated is, however, less pronounced for packings obtained for high degrees of freedom in particle motions. This discrepancy indicates that high values for the parameters $A_{\text {swiv }}^{\max }$ and $A_{\text {slide }}^{\max }$ allows for the effect of $\theta_{\text {ini }}$ on final packing porosities to be limited.

\section{Effect of disk aspect ratio}

The relation between particle aspect ratio and packing porosity has been previously studied for disks using either experimental [41-43] or 3D numerical procedures [29,38,44,45]. As shown in Fig. 5(a), all results reveal a U-shaped relationship between porosity and aspect ratio, where an $\mathcal{R}$ value of 1 represents the minimum of the curve. In the present section, we focus on flat disks with $R<1$ and extend the investigation for low aspect ratio values down to 0.01 to approach the anisotropy
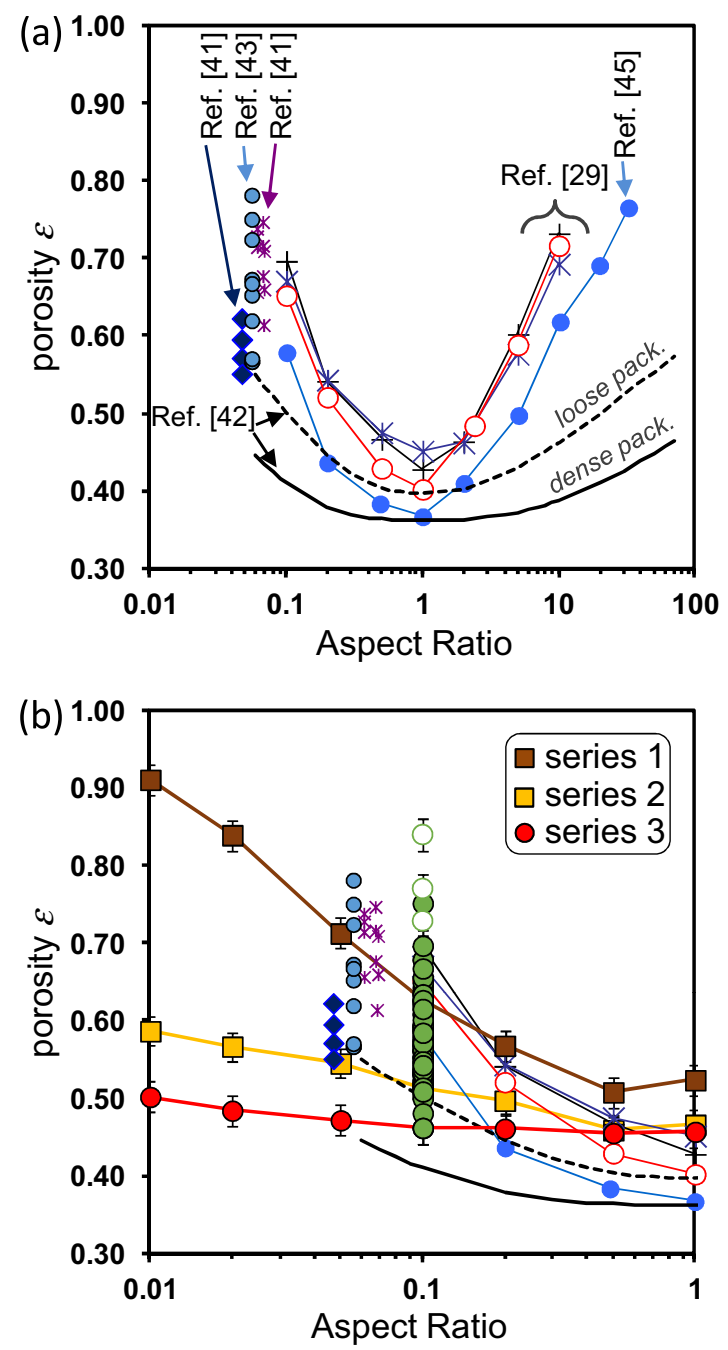

FIG. 5. (Color online) Effect of disk aspect ratio on the packing porosity $\varepsilon$. (a) Overview of existing simulation $[29,45]$ and experimental data [41-43], including porosity measurements for natural kaolinite [43] and mica [41] clay powders. (b) Comparison between existing data and results obtained in the present study for the three series of calculations using the parameters reported in Table II. For aspect ratio $\mathcal{R}=0.1$, all packing porosities shown in Fig. 4 are also reported as green circles, whereas open ones correspond to packings in which the presence of large pores, as shown in Fig. 3(c), is detected.

of clay particles. Three series of calculations are performed depending on the degree of freedom given to particle motions and the initial angles $\theta_{\text {ini }}$ generated using the algorithm parameters reported in Table II. For the first two series, angles $\theta_{\text {ini }}$ are randomly generated over the range $0<\theta_{\text {ini }}<\pi / 2$, but these two sets of simulations differ with respect to the degree of freedom given to particle motions. The same sets of maximum amplitude parameters discussed in Sec. III B, i.e., $A_{\text {swiv }}^{\max }=10^{\circ}$, $A_{\text {slide }}^{\max }=\tilde{d} / 7$ and $A_{\text {swiv }}^{\max }=80^{\circ}, A_{\text {slide }}^{\max }=5 \tilde{d}$, are thus considered for low and high degrees of freedom, respectively. For the third series, we consider the set of parameters leading to the lowest porosity in the tests performed in Sec. III B, i.e., an initial orientational configuration of particles with $\theta_{\text {ini }}=0$ and maximum amplitude parameters leading to a high degree of freedom in motion (i.e., $A_{\text {swiv }}^{\max }=80^{\circ}$ and $A_{\text {slide }}^{\max }=5 \tilde{d}$ ). Due 
TABLE II. Algorithm parameters used for the three series of calculations. The parameters $N_{p}, \tilde{d}, w, d_{\text {cont }}$, and $n_{s p}$ represent the number of particles, the mean diameter of a circle with an equivalent basal surface, the width of the simulation box, the characteristic contact distance along the $z$ axis, and the number of scan points on the bottom basal surface of the settling particle, respectively. Each settling particle is introduced into the simulation box with an initial angular value relative to the existing bed $\theta_{\text {ini }}$ and with maximum amplitude parameters for swivel, rotation, and slide motions (i.e., $A_{\text {swiv }}^{\max }, A_{\text {rot }}^{\max }$, and $A_{\text {slide }}^{\max }$, respectively).

\begin{tabular}{lccc}
\hline \hline & \multicolumn{3}{c}{ Calculation series } \\
\cline { 2 - 4 } Parameter & 1 & 2 & 3 \\
\hline$N_{p}$ & 2500 & 2500 & 2500 \\
$w$ & $7.5 \tilde{d}$ & $7.5 \tilde{d}$ & $7.5 \tilde{d}$ \\
$d_{\text {cont }}$ & $5 \times 10^{-4} \tilde{d}$ & $5 \times 10^{-4} \tilde{d}$ & $5 \times 10^{-4} \tilde{d}$ \\
$n_{s p}$ & 150 & 150 & 150 \\
$\theta_{\text {ini }}$ & $\theta_{\text {ini }} \in\left(0, \frac{\pi}{2}\right)$ & $\theta_{\text {ini }} \in\left(0, \frac{\pi}{2}\right)$ & $\theta_{\text {ini }}=0$ \\
$A_{\text {swiv }}^{\max }$ & $10^{\circ}$ & $80^{\circ}$ & $80^{\circ}$ \\
$A_{\text {rot }}^{\max }$ & $10^{\circ}$ & $80^{\circ}$ & $80^{\circ}$ \\
$A_{\text {slide }}^{\max }$ & $\tilde{d} / 7$ & $5 \tilde{d}$ & $5 \tilde{d}$ \\
\hline \hline
\end{tabular}

to the circular basal shape of the particle, in-plane rotation motions are not considered. As shown in Fig. 5(b), the three calculations yield a U-shaped curve, but the obtained porosity values are significantly different. Indeed, for a given aspect ratio value, the obtained packing porosities logically decrease when increasing the degree of freedom in particle motion and when decreasing the values of $\theta_{\text {ini. }}$. It should be noted that the difference in porosity resulting from the use of different sets of parameters diminishes with increasing particle aspect ratio. This behavior is fully consistent with a low particle dimensional anisotropy, which tends to minimize the effects of both the degree of freedom of particle motions and the initial particle configuration.

\section{Comparison with existing algorithms and experiments}

The sensitivity tests on the algorithm parameters for flat disks with $R=0.1$ show that a wide range of porosities can be obtained depending on both the degree of freedom given to particle motions and the initial orientational configuration of the particle. The obtained porosity values range between 0.46 and 0.84 , although for porosity values greater than 0.7 , large pores are systematically detected. As illustrated in Fig. 5(b), these results are consistent with the simulated packing porosities of Coelho et al. [29] and the experimental packings of Zou and $\mathrm{Yu}$ [42]. It should be pointed out, however, that due to the lack of collective rearrangements of particles already deposited, the sequential deposition algorithm cannot lead to extremely dense packing as obtained when considering collective motions of particles or particle expansion methods (see, for example, [46-50]). As an example, the experimental work of Zou and $\mathrm{Yu}$ [42] on a disk having an aspect ratio similar to that used here showed that a porosity value of 0.4 (referred to as dense packing by these authors) was obtained from an original packing porosity of $\sim 0.5$ (i.e., loose packing) using a vertical tapping method until no change of volume was detected, a technique strongly favoring collective particle rearrangements. Despite the lack of collective motions, the porosity around 0.46 is satisfactory when compared to this experimental work. Still, porosity values do not allow for a direct comparison between different algorithms if the anisotropic organization of the obtained porous media is not considered. Information about the degree of anisotropy of particle orientation in a packing and the main symmetry axis of porous media can be obtained by analyzing the $Q$-tensor order parameter [51,52]. For $N$ particles, the $Q$ tensor is a symmetric traceless second-rank tensor constructed from the unit vector $v^{\alpha}$ pointing perpendicular to each particle at a position $x^{\alpha}$ as follows:

$$
Q_{i j}=\frac{V}{N} \sum_{\alpha}\left(v_{i}^{\alpha} v_{j}^{\alpha}-\frac{1}{3} \delta_{i j}\right) \delta\left(x-x^{\alpha}\right) .
$$

This $Q$ tensor can also be written in terms of a scalar order parameter $S$, a biaxiality parameter $\eta$, and three eigenvectors $\bar{n}, \bar{k}$ and $\bar{l}$ as follows:

$$
Q_{i j}=\frac{S}{2}\left(3 l_{i} l_{j}-\delta_{i j}\right)+\eta\left(k_{i} k_{j}-n_{i} n_{j}\right) .
$$

The order parameter $S$ ranges from 0 for isotropic systems to 1 for perfectly oriented particles, whereas the biaxiality parameter $\eta$ indicates the presence $(\eta \neq 0)$ or the absence $(\eta=$ 0 ) of a second symmetry axis in the system. In an oriented powder, in which particles align their normal unit vector along the macroscopic average direction $\bar{l}$, the parameters $S$ and $\eta$ can both be obtained by analyzing the eigenvalues along the diagonal of the $Q$ tensor as follows:

$$
Q=\left(\begin{array}{ccc}
-\frac{S}{2}+\eta & 0 & 0 \\
0 & -\frac{S}{2}-\eta & 0 \\
0 & 0 & S
\end{array}\right) .
$$

The analysis of the obtained eigenvalues allows for the assessment of the degree of biaxiality of the obtained packings. For all packings of disks with $\mathcal{R}=0.1$, the mean $\eta$ value is $\sim 0.003$, indicating a negligible degree of biaxiality. The obtained porous media can thus be considered to display uniaxial symmetry along the eigenvector $\bar{l}$ of the $Q$ tensor. In addition, calculation of the mean value for the angle $\beta$ between the eigenvector $\bar{l}$ and the $z$ axis of the simulation boxes yields a value of $0.47^{\circ}$. This result indicates that the $z$ axis can be considered the sole symmetry axis of the porous media. According to the notation used in the present study, the scalar order parameter for particles of the same size $S_{\text {part }}^{\text {calc }}$ can also be defined based on the average of the second-order Legendre polynomial as follows:

$$
S_{\text {part }}^{\text {calc }}=\left\langle 3 \cos ^{2} \theta-1\right\rangle / 2,
$$

where $\theta$ represents the angle between the normal unit vector of the particle and the $z$ axis of the simulation box as defined in Sec. II B.

The resulting porosities are plotted as a function of the order parameter $S_{\text {part }}^{\text {calc }}$ in Fig. 6(a). Except for the pathological packings with large pores discussed in Sec. III A, all porosity values fall onto a single master curve when plotted as a function of $S_{\text {part }}^{\text {calc }}$. This result demonstrates that regardless of the degree of freedom given to particle motions or the initial configuration 
(a)

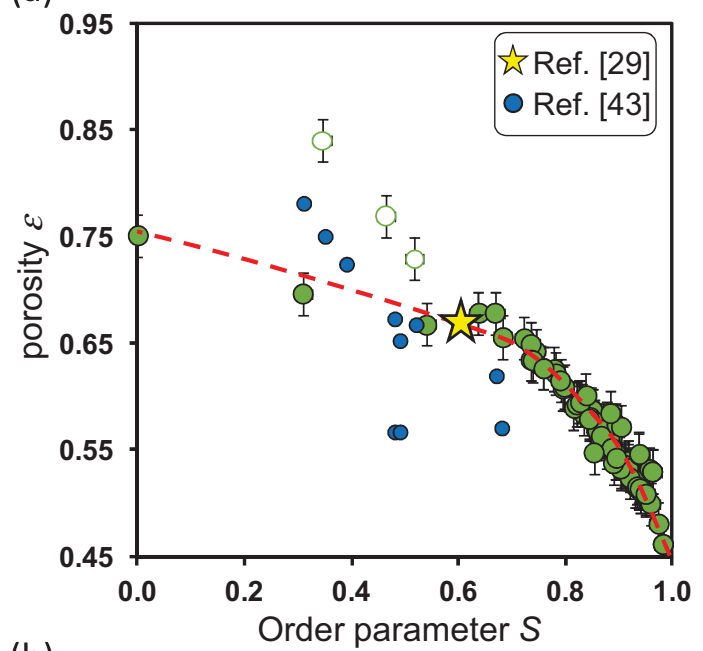

(b)

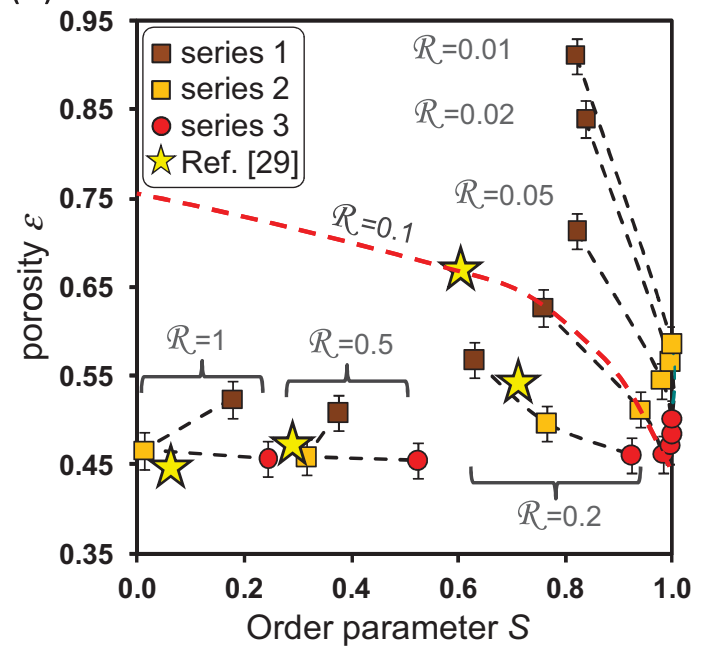

FIG. 6. (Color online) Evolution of porosity $\varepsilon$ with the scalar order parameter of the packing $S$. (a) All data for disks with $\mathcal{R}=0.1$ shown in Fig. 4. Open circles correspond to packings in which the presence of large pores, as shown in Fig. 3(c), is detected. The obtained data are compared to the packing obtained by Coelho et al. [29] using a similar algorithm and to those obtained for natural kaolinite powder $(\mathcal{R}=0.06)$ by Perdigon-Aller et al. [43]. The dotted curve is plotted as a guideline. (b) Same plot for disk particles with the different aspect ratios shown in Fig. 5.

for the first contact of the particle on the bed, a porosity value is systematically associated with a given anisotropy in particle orientation. Furthermore, such a relation allows for a comparison of the different algorithms. Indeed, the packing obtained by Coelho et al. [29] for the same disk aspect ratio lies perfectly on this curve, indicating a consistency of our algorithm with the one proposed by these authors, both of which are based on the sequential particle deposition procedure. In addition, it should be noted that the experimental data of Perdigon-Aller et al. [43] can also be placed on this porosity vs $S_{\text {part }}^{\text {calc }}$ plot. These latter data were obtained for a polydisperse powder of kaolinite particles with a mean aspect ratio of 0.06 submitted to different degrees of compaction. As shown in Fig. 6(a), despite both the polydisperse nature of particles in the kaolinite powder and the possible presence of macroporosity in these reconstructed natural samples, the porosity values are on the same order as those obtained in the present numerical study.

The results displayed in Fig. 5(b) for particles with varying aspect ratios additionally indicate that the present algorithm provides consistent porosity values compared to existing experimental or simulation data. Indeed, experimental porosities for Millar disks [41] as well as for kaolinite [43] and mica [41] powders are satisfactorily reproduced using contrasting degrees of freedom in particle motions and $\theta_{\text {ini }}$ values. Moreover, it can be observed that even if the aspect ratio of particles was not measured, the porosity values at 0.6-0.65 obtained by Mammar et al. [53] for mica and montmorillonite powders also fall within the range of porosity values derived in the present study. Calculations performed by assuming low degrees of freedom appear to lead to slightly higher porosities when compared with those performed using other existing algorithms for $\mathcal{R}>0.2$. Such higher porosities likely result from the limited amplitude given to swivel motions (i.e., $A_{\text {swiv }}^{\max }=10^{\circ}$ ), which does not allow particles with high $\theta_{\text {ini }}$ angles to pivot and thereby lie flat on the particle bed. For all series, the porosities obtained for $\mathcal{R}=1$ are slightly higher than those obtained in previous studies, possibly indicating a somewhat lower efficiency of our algorithm in detecting contact points on particle edges. However, this difference remains marginal, and in the case of clay particles that usually display aspect ratios not higher than 0.3 [34], the obtained results reflect the robustness of our algorithm in predicting correct packing properties. The extension of $R$ values down to 0.01 in the present study highlights the effect of particle anisotropy on porosity values. Indeed, porosity values obtained for anisotropic particle geometries are observed to be strongly dependent on the degree of freedom in particle motion and on the initial orientation of the particles. In addition, as pointed out by Coelho et al. [29], such an effect is likely to be enhanced by the sharp-edged morphology of the particles. This latter effect is consistent with the evolution of porosity as a function of $\mathcal{R}$ values for ellipsoids observed by these authors; specifically, the authors observed a limited evolution for packings obtained with high values of amplitude parameters and $\theta_{\text {ini }}=0$. In Fig. 6(b) the dependence of the porosity on $S_{\text {part }}^{\text {calc }}$ is plotted and compared with the data reported by Coelho et al. [29] for different disk aspect ratios. For disks with $\mathcal{R}=0.1$, the porosity values obtained for the different aspect ratios in the present study are consistent with those obtained by Coelho et al. [29]. It should be noted that, as previously mentioned, the effect of the limited amplitude given to swivel and slide motions is indicated for disk aspect ratios of 0.5 and 1.0 by the slightly higher porosities for a given $S_{\text {part }}^{\text {calc }}$ value obtained for these two packings. Finally, these results illustrate the significant effect of particle aspect ratio and likely particle shape on the obtained $\varepsilon$ vs $S_{\text {part }}^{\text {calc }}$ curves.

\section{EFFECT OF PARTICLE $\ell_{a} / \ell_{b}$ RATIO AND POLYDISPERSITY}

In this section, we investigate the effects of different particle geometry parameters on the porosity of the packings obtained using the sequential deposition algorithm, previously validated for disks in Sec. III D. The first parameter considered 
is the $\ell_{a} / \ell_{b}$ ratio of elliptic disks, which, to the authors' knowledge, has never been investigated for 3D packings. We will then focus on the effect of polydispersity in particle size, $\ell_{a} / \ell_{b}$ and aspect ratio because these parameters are highly relevant to studying natural porous media, as illustrated by the experimental data reported in Table I. In line with the analysis performed in Sec. III C, the same three series of calculations are performed using the sets of parameters reported in Table II. For these calculations, the maximum amplitude given to in-plane rotation motions $A_{\text {rot }}^{\max }$ is set equal to the parameter $A_{\text {swiv }}^{\max }$ (Table II).

\section{A. Influence of particle $\ell_{a} / \ell_{b}$ ratio}

The evolution of porosity with $\ell_{a} / \ell_{b}$ is shown in Fig. 7. All simulations are performed for particles with an $\mathcal{R}$ value of 0.1 . The obtained results show that the elliptic nature of particles has a negligible effect on the obtained porosity for $\ell_{a} / \ell_{b}<1.5$, whereas for higher $\ell_{a} / \ell_{b}$ values the porosity of the obtained packing increases significantly. This result
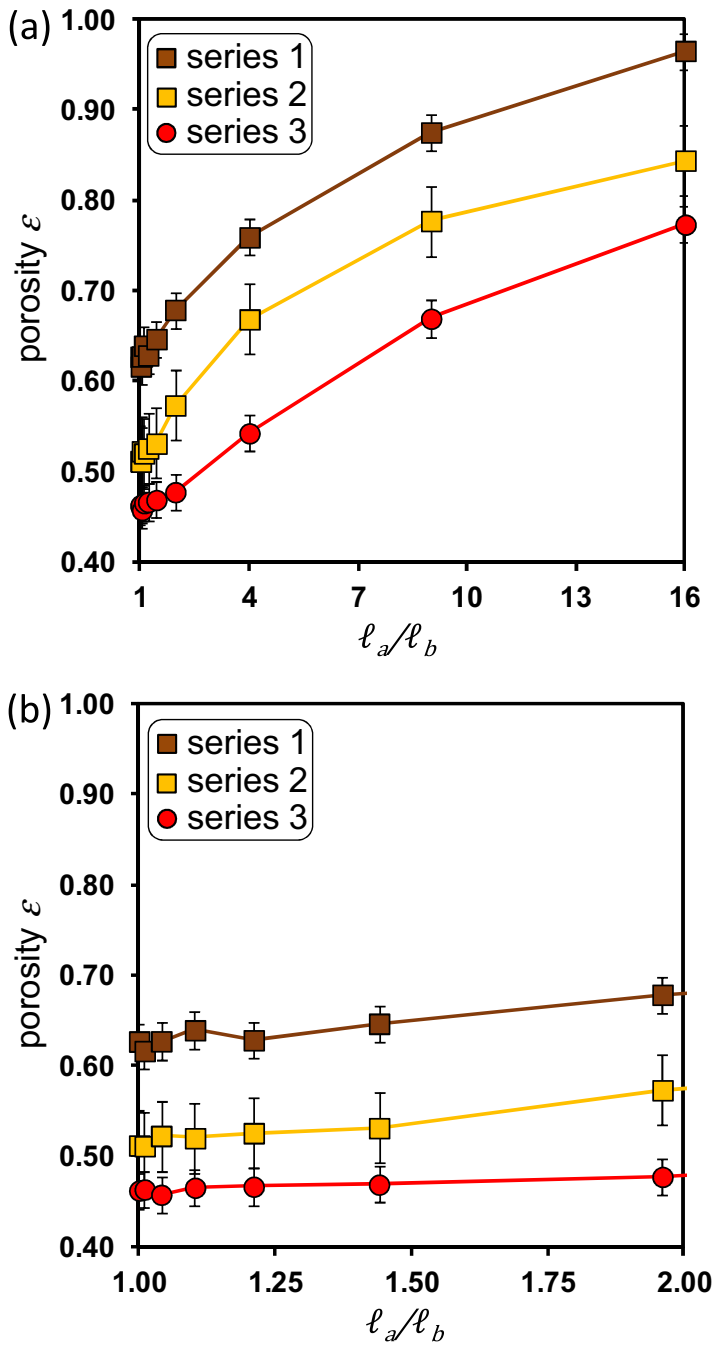

FIG. 7. (Color online) (a) Evolution of porosity $\varepsilon$ as a function of the ratio $\ell_{a} / \ell_{b}$ for the three series of calculations performed using the parameters reported in Table II. (b) Same graph, zoomed in the $1<\ell_{a} / \ell_{b}<2$ range. is similar to the evolution obtained for prolate particles for $\mathcal{R}>1[29,42,45]$ shown in Fig. 5(a). Furthermore, these results are fully consistent with the $2 \mathrm{D}$ simulations performed by Rothenburg and Bathurst [35] and Delaney et al. [36], who reported an increase in porosity for $\ell_{a} / \ell_{b}>2$.

\section{B. Effect of polydispersity in particle size and shape}

The effects of polydispersity in particle $\ell_{a} / \ell_{b}$ ratio, aspect ratio and size on the packing porosities are reported in Fig. 8 for the three calculation series detailed in Table II. For all series, the $\sigma$ value of the lognormal distribution was varied from 0 to 0.8 , i.e., a range of values relevant to natural samples (Table I).

Fig. 8(a) shows the effect of particle aspect ratio polydispersity on the porosity of particles with a mean aspect ratio of 0.1 and $a \ell_{a} / \ell_{b}$ ratio of 1 . For series 1 , no significant trend can be observed, whereas for series 2 and 3 , an increase in $\sigma$ leads to a slight increase in porosity. This behavior is likely related to the high anisotropy of the packings obtained using a high degree of freedom for particle motions. Indeed, for such packings, the dispersity in the particle aspect ratio likely enhances the corrugation of the particle bed surface. This feature in turn limits the ability of the settling particle to explore more favorable configurations and induces a small rise in the final porosity.

The effect of dispersity in the particle $\ell_{a} / \ell_{b}$ ratio on final porosities is reported in Fig. 8(b). The simulation parameters correspond to particles with $\mathcal{R}=0.1$ and a median value for the lognormal distribution of $\ell_{a} / \ell_{b}$ ratio set to 1.44 . This latter value is chosen as an average of the different $\ell_{a} / \ell_{b}$ median values obtained for the different fractions in natural samples (Table I). For the three simulation series, an increase in packing porosity is observed with the standard deviation of the lognormal distribution. Such a steady increase in porosity is consistent with the results obtained in Sec. IV A. Indeed, for packings of particles with the same $\ell_{a} / \ell_{b}$ ratio, a rise in porosity is observed for $\ell_{a} / \ell_{b}>1.5$, whereas below this threshold value, the packings display similar porosities (Fig. 7). As a logical effect of the lognormal distribution of $\ell_{a} / \ell_{b}$, an increase in standard deviation induces an increase in the number of particles with $\ell_{a} / \ell_{b}>1.5$, likely contributing to the increase in the packing porosities for all calculation series observed in Fig. 8(b).

Using a sequential deposition algorithm and a lognormal distribution in particle size, Mourzenko et al. [31] reported a decrease in porosity from 0.41 to 0.35 for polydisperse spheres when increasing the lognormal standard deviation from 0 to 0.6 . In the present work, the obtained packings display behavior similar to that shown in Fig. 8(c). For the three calculation series, the simulation parameters correspond to disks with $R=0.1, \ell_{a} / \ell_{b}=1$ and a median value for the distribution on $\tilde{d}$. Owing to the large particles that must be included in the simulation for large $\sigma$ values, the size of the simulation box and the number of settling particles are increased to $w=15$ and 3200 particles, respectively. As shown in Fig. 8(c), all packings display a decrease in porosity with increasing polydispersity, except for the packing obtained for series 1 with $\sigma=0.8$. In this latter case, the high size dispersion coupled with a low degree of freedom in particle 

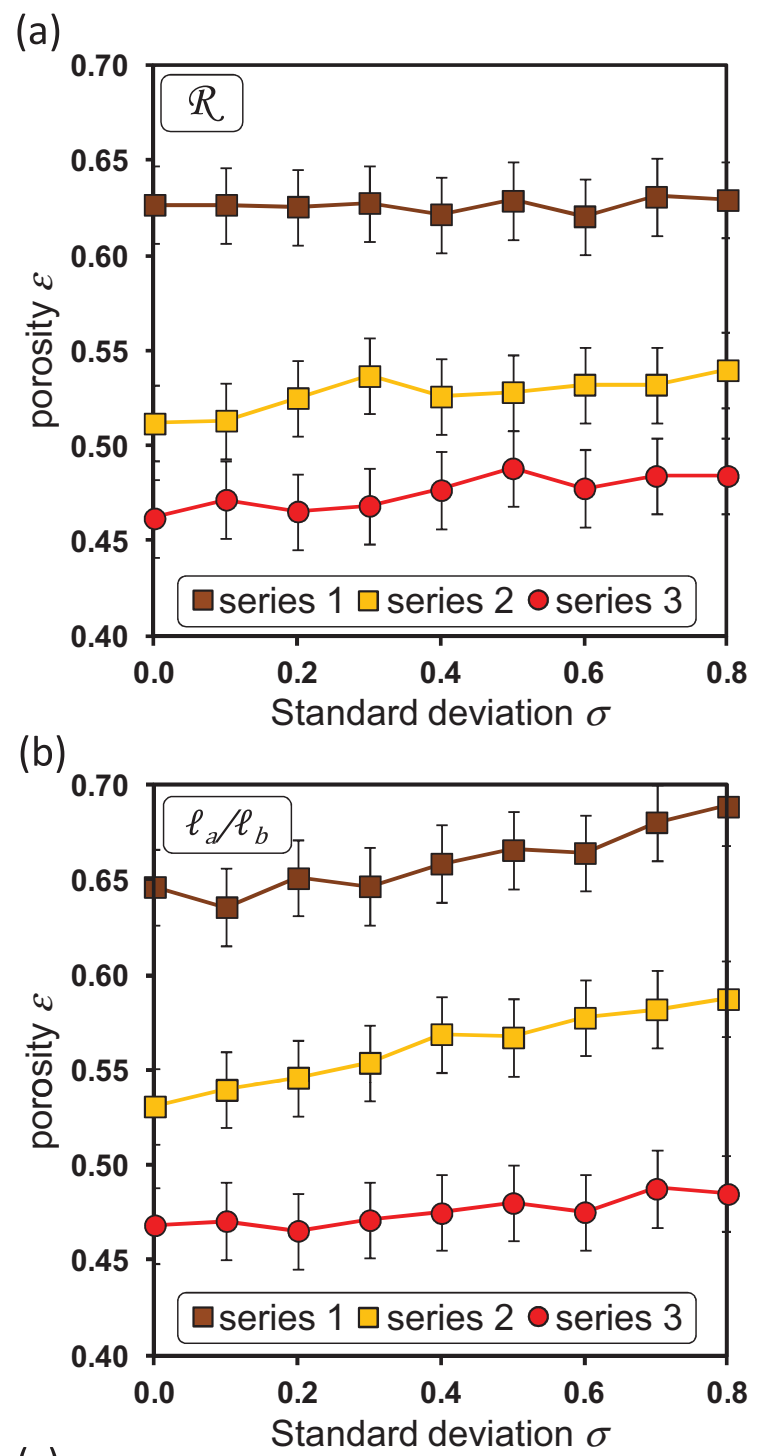

(c)

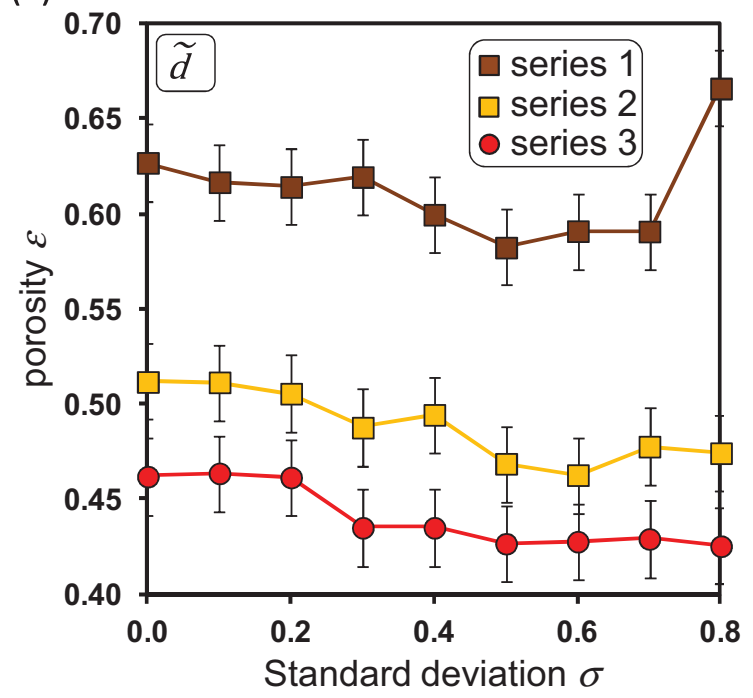

FIG. 8. (Color online) Effect of the standard deviation $\sigma$ of the $\log$-normal distribution on the packing porosities $\varepsilon$ for the three series of calculations performed using the parameters reported in Table II. The log-normal distribution applied to (a) aspect ratio $\mathcal{R}$, (b) particle ratio $\ell_{a} / \ell_{b}$, and (c) disk diameter $\tilde{d}$.
TABLE III. Algorithm parameters for the simulation of clay porous media composed of natural vermiculite size fractions. The notation is the same as in Table II. The parameters $w, d_{\text {cont }}$, and $A_{\text {slide }}^{\max }$ are reported in $\mu \mathrm{m}$.

\begin{tabular}{lccc}
\hline \hline Parameter & $0.1-0.2 \mu \mathrm{m}$ & $1-2 \mu \mathrm{m}$ & $10-20 \mu \mathrm{m}$ \\
\hline$N_{p}$ & 10000 & 8000 & 2200 \\
$w$ & 1.2 & 6 & 200 \\
$d_{\text {cont }}$ & 0.001 & 0.001 & 0.01 \\
$n_{s p}$ & 150 & 150 & 500 \\
$\theta_{\text {ini }}$ & $60^{\circ}$ & $80^{\circ}$ & $85^{\circ}$ \\
$A_{\text {swiv }}^{\max }$ & $40^{\circ}$ & $20^{\circ}$ & $20^{\circ}$ \\
$A_{\text {rot }}^{\max }$ & $40^{\circ}$ & $20^{\circ}$ & $20^{\circ}$ \\
$A_{\text {slide }}^{\max }$ & 0.01 & 0.1 & 2 \\
\hline \hline
\end{tabular}

motion generates large pores in the particle bed, which in turn increases the porosity. It is worth noting that an increase in $\sigma$ from 0 to 0.6 leads to a decrease in porosity of $\sim 0.04$, i.e., a value similar to the decrease of 0.06 obtained for polydisperse spheres [31].

\section{APPLICATION TO NATURAL CLAY POROUS MEDIA}

The purpose of this section is to apply the sequential deposition algorithm to mimic particle organization in clay porous media built using three different size fractions of natural vermiculite powders. The parameters used in these simulations are reported in Table III. The geometrical and orientational properties of particles in the obtained packings are then compared with experimental data derived for the same samples. Finally, these virtual 3D porous media are used to derive information about the anisotropy of the pore and solid phases in the samples.

\section{A. Simulation details and representative elementary volume analysis}

The width $w$ of the simulation boxes considered for the $0.1-0.2,1-2$, and 10-20 $\mu \mathrm{m}$ size fractions are $w \sim 14.8 \tilde{d}$, $w \sim 14.3 \tilde{d}$, and $w \sim 8.2 \tilde{d}$, respectively. The characteristic contact distance along the $z$ axis, $d_{\text {cont }}$, is set to $1 \mathrm{~nm}$, whereas the number of scan points $n_{s p}$ on the bottom basal surface of the settling particle is set to 150 for both the $0.1-0.2$ and $1-2 \mu \mathrm{m}$ size fractions. To (i) favor the detection of contact points despite their large dimensions and (ii) limit the calculation time, particles in the 10-20 $\mu \mathrm{m}$ size fractions are assigned $n_{s p}$ and $d_{\text {cont }}$ values of 500 and $10 \mathrm{~nm}$, respectively (Table III). The numbers of particles $N_{p}$ considered for the $0.1-0.2,1-2$, and 10-20 $\mu \mathrm{m}$ size fractions are 10000,8000 , and 2200, respectively, initially introduced with a fixed $\theta_{\text {ini }}$ angle and with the particle basal surface, aspect ratio, and ratio $\ell_{a} / \ell_{b}$ defined based on the log-normal distribution parameters reported in Table I. Finally, the maximum amplitude parameters for both swivel or in-plane rotational motions as well as $A_{\text {slide }}^{\max }$ are reported in Table III. These parameters were chosen using a trial-and-error procedure, i.e., different packings were generated and the porous medium fitting at best the experimental scalar order parameter $S^{\text {expt }}$, reported by Hubert et al. [32], was retained for further analysis. 


\section{B. Comparison with experimental data}

The particle packings obtained for the different size fractions using the calculation parameters and particle morphological describers reported in Tables III and I, respectively, are displayed in Fig. 9(a). For all samples, the analysis of the $Q$ tensor using Eq. (2) reveals $\eta$ and $\beta$ values below $\sim 0.01$ and $1.2^{\circ}$, respectively, indicating that the $z$ axis of the simulation box is the sole symmetry axis of the system (Table IV). Figure 9(b) presents profiles of $\varepsilon$ and $S_{\text {part }}^{\text {calc }}$ along the $z$ axis of the simulation boxes. This latter profile is obtained in the same way as the $\varepsilon$ profile, i.e., by calculating, for each slice along the $z$ axis of thickness $d z$ the local $S_{\text {part }}^{\text {calc }}$ value using Eq. (4) for particles with barycenter altitude $r_{z}$ in the range $z<r_{z} \leqslant z+d z$.

Rather than considering the mean value of $S_{\text {part }}^{\text {calc }}$ from the linear part of the profile, the full distribution functions of particle orientation in the different vermiculite fractions can be extracted to directly compare with the experimental rocking

TABLE IV. Analysis of the virtual porous media obtained for the different size fractions of vermiculite and comparison with experimental data. The parameters $\eta$ and $\beta$ (in degrees) represent the degree of biaxiality and the angle between the eigenvector $\bar{l}$ and the $z$ axis of the simulation box, respectively. The minimum and maximum altitudes (i.e., $z_{\min }$ and $z_{\max }$, respectively, in $\mu \mathrm{m}$ ) are used to calculate the geometrical properties of the packings, such as the porosity $\varepsilon$. The parameters $l_{v}(\mu \mathrm{m})$ and $f_{o}$ correspond to the resolution of the $512^{3}$ voxelized image and the fraction of particle overlap, respectively. The experimental scalar order parameter $S^{\text {expt }}$ derived from Hubert et al. [32] is compared to the calculated values either unweighted $\left(S_{\text {part }}^{\text {calc }}\right)$ or weighted by the volume of individual particles $\left(S_{\text {vol }}^{\text {calc }}\right)$. The calculated ensemble average lateral $A_{L}^{\text {calc }}$, basal $A_{B}^{\text {calc }}$, and total $A_{T}^{\text {calc }}$ surface area values (in $\mathrm{m}^{2} / \mathrm{g}$ ) are compared to the experimental values $\left(A_{L}^{\text {expt }}, A_{B}^{\text {expt }}\right.$, and $A_{T}^{\text {expt }}$, respectively, reported by Reinholdt et al. [34]. Here $A_{B \text { cont }}^{\text {calc }}$ (in $\mathrm{m}^{2} / \mathrm{g}$ ) represents the overall amount of basal surface involved in the contact between particles. The sizes of representative elementary volumes for porosity $\left(w_{\varepsilon}\right)$ and solid-pore interface ( $w_{s-p \text { interf }}$ ) are reported in $\mu \mathrm{m}$. Here $R_{p}$ and $R_{s}$ represent the ratios of the mean chord length momentum between the $z$ and $x y$ directions for the pore and solid phases, respectively.

\begin{tabular}{lccc}
\hline \hline Parameter & $0.1-0.2 \mu \mathrm{m}$ & $1-2 \mu \mathrm{m}$ & $10-20 \mu \mathrm{m}$ \\
\hline$\eta$ & 0.007 & 0.008 & 0.011 \\
$\beta$ & 0.12 & 0.32 & 1.19 \\
$z_{\min } / z_{\max }(\mu \mathrm{m})$ & $0.15 / 1.35$ & $1 / 7$ & $7 / 207$ \\
$\varepsilon$ & 0.63 & 0.64 & 0.70 \\
$l_{v}(\mu \mathrm{m})$ & $2.3 \times 10^{-3}$ & $1.2 \times 10^{-2}$ & 0.4 \\
$f_{o}$ & $6.9 \times 10^{-5}$ & $2.3 \times 10^{-5}$ & $2.0 \times 10^{-6}$ \\
$S^{\text {expt }}$ & 0.66 & 0.36 & 0.10 \\
$S_{\text {part }}^{\text {calc }}$ & 0.63 & 0.36 & 0.10 \\
$S_{\mathrm{vol}}^{\text {calc }}$ & 0.60 & 0.34 & 0.11 \\
$A_{L}^{\text {calc }} / A_{L}^{\text {expt }}$ & $18.3 / 18.0$ & $2.6 / 3.0$ & $0.07 / 0.07$ \\
$A_{B}^{\text {calc }} / A_{B}^{\text {expt }}$ & $76.2 / 76.2$ & $10.3 / 11.7$ & $0.32 / 0.37$ \\
$A_{T}^{\text {calc }} / A_{T}^{\text {expt }}$ & $94.5 / 94.2$ & $12.9 / 14.7$ & $0.39 / 0.44$ \\
$A_{B \text { cont }}^{\text {calc }}$ & 5.3 & 0.66 & 0.014 \\
$A_{B \text { cont }}^{\text {calc }} / A_{B}^{\text {calc }}$ & $6.9 \%$ & $6.4 \%$ & $4.3 \%$ \\
$w_{\varepsilon}$ & 0.39 & 4.2 & 87 \\
$w_{s-p \text { interf }}$ & 0.66 & 4.8 & 112 \\
$R_{p}$ & 0.494 & 0.689 & 0.880 \\
$R_{s}$ & 0.481 & 0.691 & 0.916 \\
\hline \hline
\end{tabular}

curves studied in detail by Hubert et al. [32]. To this end, let us consider the orientation distribution function relative to the $z$ axis of particle volumes $F_{v}(\theta)$. This volume correction is performed to account for contrasting particle sizes that may not give rise to the same orientation properties. This function is thus defined as follows:

$$
\int_{0}^{\pi} F_{v}(\theta) d \theta=1 .
$$

The calculated intensity $I_{c}(\theta)$ to be compared to experimental RC curves $I_{e}(\theta)$ simply requires accounting for the solid angle correction, considering the uniaxial symmetry of the system, as follows:

$$
I_{c}(\theta)=\frac{A F_{v}(\theta)}{2 \pi \sin (\theta)},
$$

where $A$ is a constant for intensity renormalization corresponding to the integrated intensity of the experimental $\mathrm{RC}$ given by

$$
A=\int_{0}^{\pi} I_{e}(\theta) 2 \pi \sin (\theta) d \theta .
$$

The calculated RCs are compared with the experimental ones in Fig. 9(c) for particles with barycenter altitude $r_{z}$ found in the range $z_{\min }<r_{z} \leqslant z_{\max }$ (Table IV) without any further intensity adjustment. For the $0.1-0.2$ and $1-2 \mu \mathrm{m}$ size fractions, the consideration of particles from the linear part of the $\varepsilon$ and $S_{\text {part }}^{\text {calc }}$ profiles allows for the experimental RCs to be fully reproduced. It is worth noting that two different contributions (i.e., anisotropic and isotropic contributions) are commonly considered in the quantification of experimental RC data $[54,55]$. For these two finer fractions, the present analysis, however, shows that these two contributions result from an overall degree of anisotropy rather than from the presence of two different types of organization in the system. Interestingly, the situation is slightly different for the $10-20 \mu \mathrm{m}$ fraction. The experimental RC of this sample displays a sharp and low-intensity peak over a flat isotropic contribution. All attempts to reproduce this specific RC shape using particles extracted from the linear part of the $\varepsilon$ and $S_{\text {part }}^{\text {calc }}$ profiles proved unsuccessful irrespective of the algorithm parameters (data not shown). For this sample, it is therefore necessary to consider a nearly isotropic packing and a $z_{\text {min }}$ value slightly below the linear part of the $S_{\text {part }}^{\text {calc }}$ profile, as shown in Fig. 9(b), to reproduce the central sharp contribution on the calculated RC. This finding is particularly noteworthy because it indicates that for this poorly organized $10-20 \mu \mathrm{m}$ size fraction, the sharp central peak on the experimental RC can be assigned to the few particles lying flat on the filter holder. This interpretation is consistent with the relative proportion of this oriented contribution limited to $\sim 15 \%$ of the sample, as discussed in detail by Hubert et al. [32]. Calculated scalar order parameters either weighted (i.e., $S_{\text {vol }}^{\text {calc }}$ ) or unweighted (i.e., $S_{\text {part }}^{\text {calc }}$ ) by the particle volume for particles in the $z_{\min } \leqslant r_{z}<z_{\max }$ range for all size fractions are reported in Table IV. The similar values obtained for $S_{\mathrm{vol}}^{\text {calc }}$ and $S_{\text {part }}^{\text {calc }}$ values likely indicate that all particles display similar organizational behavior irrespective of their volume. Moreover, in line with the excellent agreement between the experimental and calculated RCs, both parameters are observed to be very close or similar to the experimental 
(a)
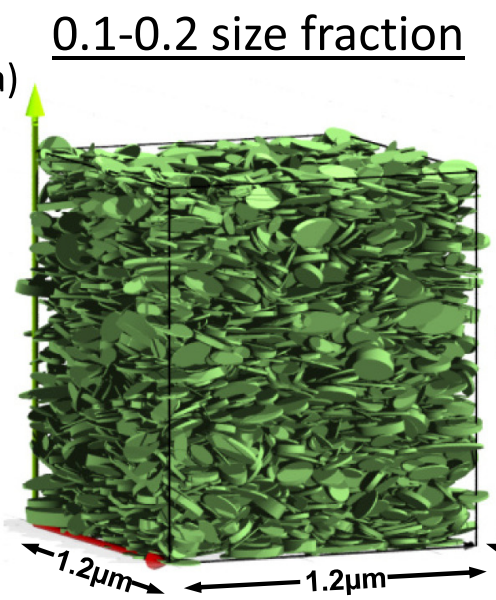

(b)

(c)
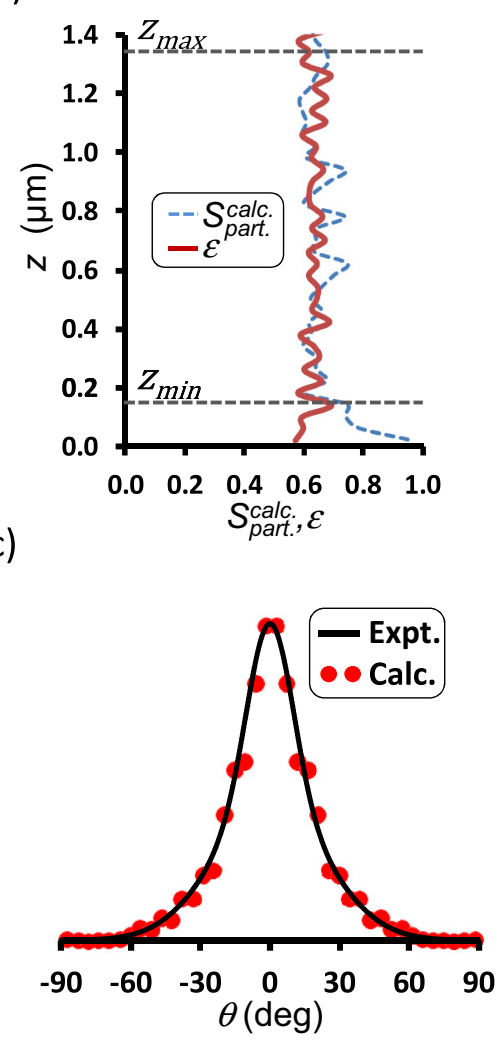

1-2 size fraction
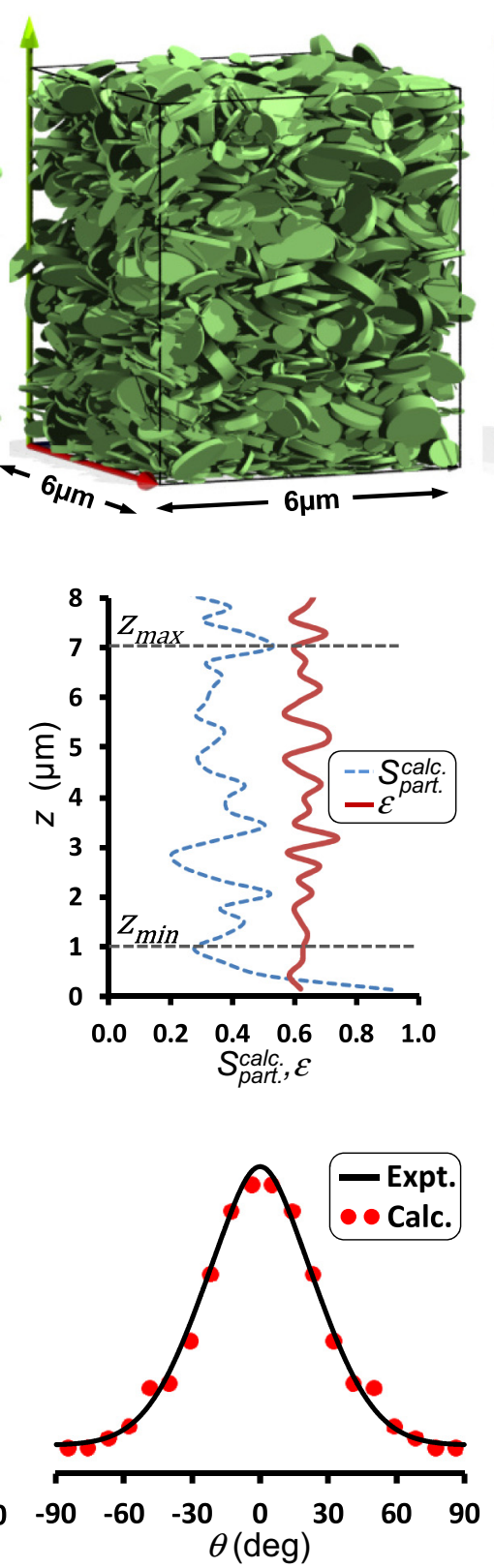

10-20 size fraction
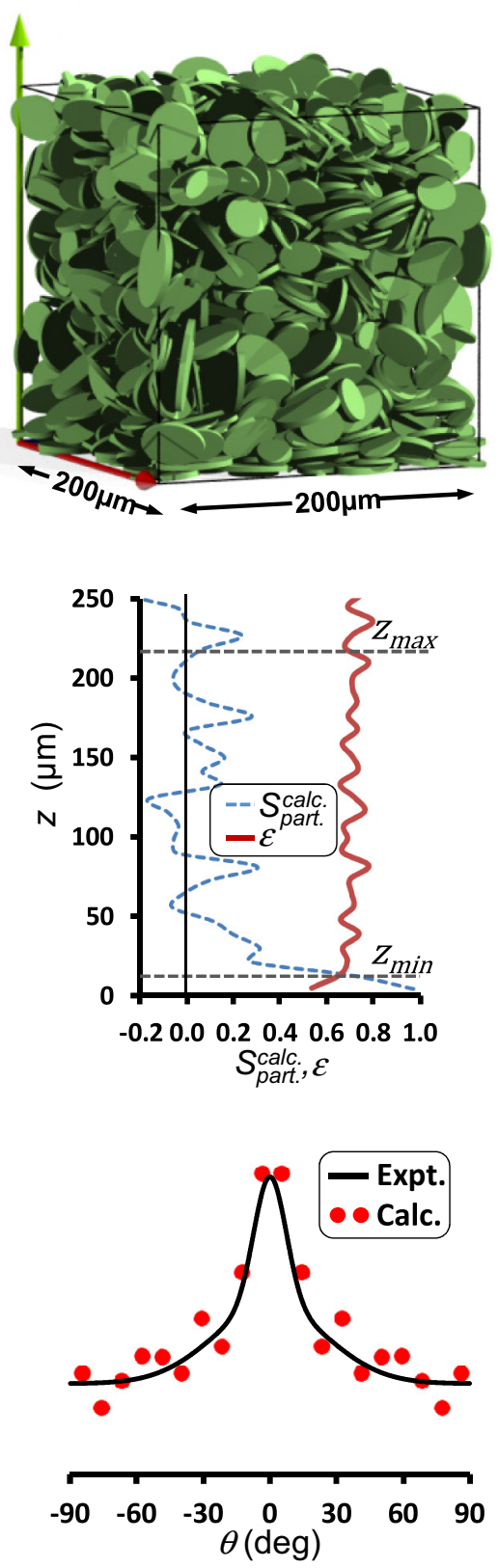

FIG. 9. (Color online) Simulation of natural swelling-clay porous media for the $0.1-0.2,1-2$, and 10-20 $\mu \mathrm{m}$ particle size fractions (from left to right). (a) Virtual 3D packings obtained using the calculation parameters reported in Table III. (b) Profiles along the $z$ direction of the porosity $\varepsilon$ and the scalar order parameter of the packing $S_{\text {part }}^{\text {calc }}$, as well as the minimum and maximum altitude limits $z_{\min }$ and $z_{\max }$ between which the properties of the porous media are extracted. (c) Comparison between experimental (solid lines) and calculated (closed circles) rocking curves.

$S^{\text {expt }}$ reported by Hubert et al. [32]. This finding likely indicates that the orientational properties of the particles in the virtual porous media shown in Fig. 9(a) are representative of our natural samples.

Direct comparison with experimental data can also be performed by comparing computed and measured particle surface areas in the porous media. Particle size and shape were generated according to log-normal distributions over the basal surface area, aspect ratio, and ratio $\ell_{a} / \ell_{b}$ parameters reported in Table I. As shown in Fig. 10, the obtained distributions of morphological describers for the generated particles logically follow experimental data, pleading for the statistical representativeness of the number of particle considered in these porous media. The good agreement between the experimental and calculated distributions should in principle guarantee the consistency of the macroscopic values for the different types of surfaces (i.e., lateral or basal surfaces). However, it should be emphasized that the calculation of this latter macroscopic quantity is only valid if the algorithm is sensitive enough to manage the settling process without the interpenetration 


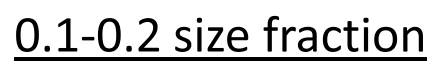

(a)

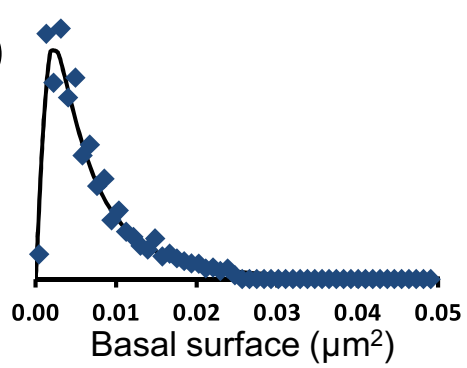

(b)
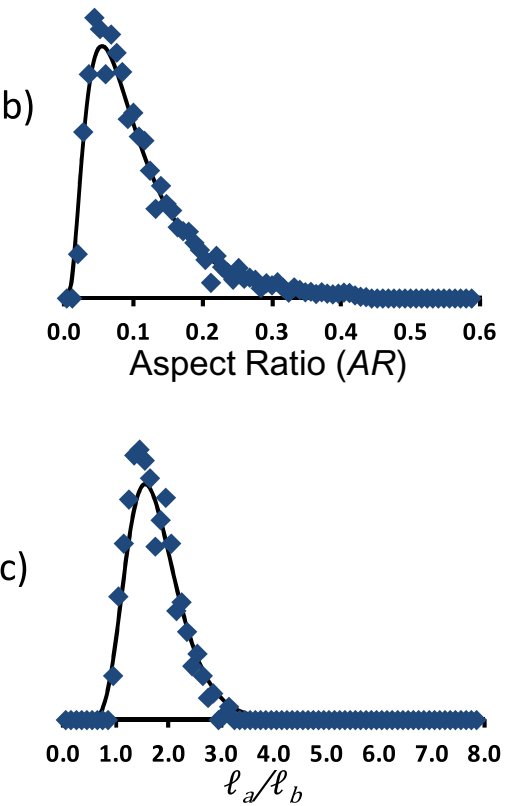

1-2 size fraction
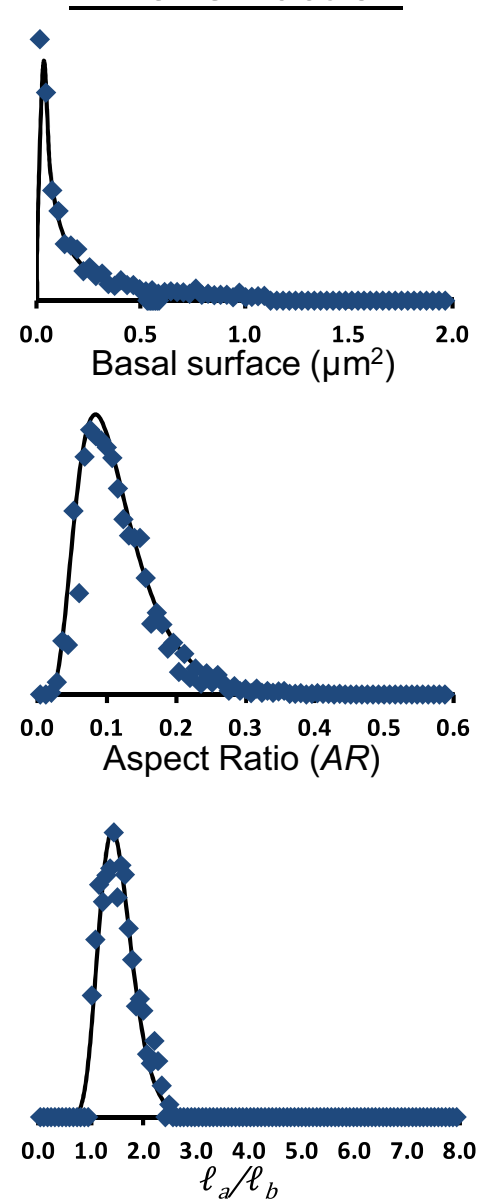

\section{0-20 size fraction}
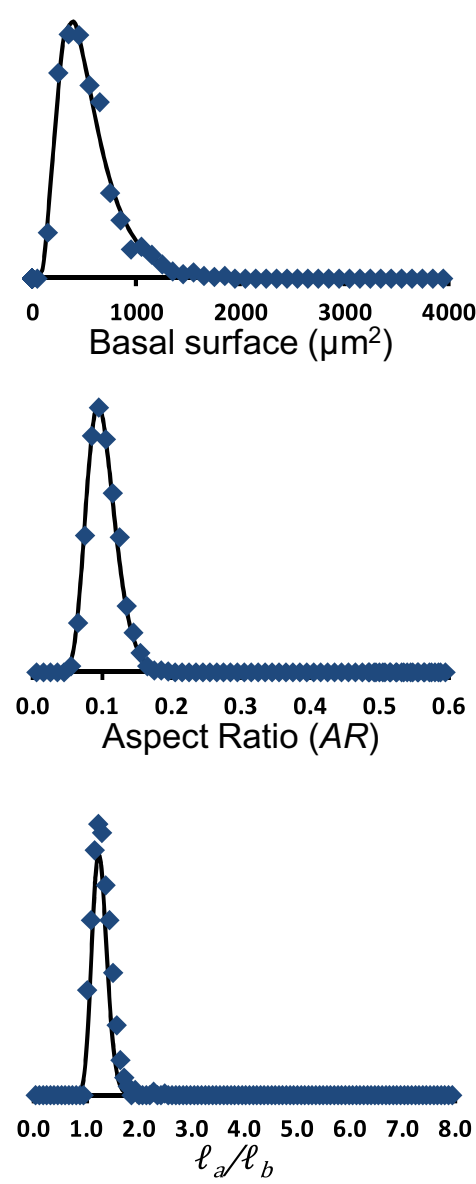

FIG. 10. (Color online) Comparison between experimental (solid lines) and calculated (closed diamonds) log-normal distributions of particle morphological parameters for the $0.1-0.2,1-2$, and $10-20 \mu \mathrm{m}$ particle size fractions (from left to right): (a) basal surface area, (b) aspect ratio, and (c) ratio $\ell_{a} / \ell_{b}$.

of particles, which would affected the surface area exposed by the solid phase. In the present study, the basal surface area of particles for a given size fraction can indeed vary by more than three orders of magnitude as shown in Fig. 10(c), and despite the computational effort undertaken to detect particle contacts (cf. Sec. II B), a limited amount of particle interpenetration may exist $[29,38]$. For this reason, the analysis of the degree of particle overlap is performed by subsampling in each porous medium a cube with the same dimension $w$ as the simulation box and composed of $512^{3}$ voxels of elementary size $l_{v}$. Each voxel volume is then either assigned to the solid phase if its center of mass is contained in one or several particle envelopes or in the pore phase otherwise [38]. The degree of particle penetration is then assessed by the relative proportion $f_{o}$ of voxels belonging to two different particles. ${ }^{1}$ The three porous media shown in Fig. 9(a) are voxelized at a resolution of $512^{3}$ in Fig. 11(a), whereas values for $f_{o}$ are reported in Table IV for the three size fractions. The obtained values for $f_{o}$ do not exceed $6.9 \times 10^{-5}$ for all size fractions, thus confirming the ability of the algorithm to

\footnotetext{
${ }^{1}$ The case in which a single voxel is shared between three particles is not addressed in this study.
}

correctly manage the interaction between particles with highly contrasting dimensional properties. Note that additional tests showed that $f_{o}$ values are found to be independent of the resolution chosen for the images (at least for resolution values $\geqslant 256^{3}$ ).

According to the negligible degree of particle overlap, the overall basal surface area $\left\langle A_{B}\right\rangle$ developed on the two flat surfaces of the elliptic disks with barycenter altitude $r_{z}$ located at $z_{\min }<r_{z} \leqslant z_{\max }$ can be calculated as follows:

$$
\left\langle A_{B}\right\rangle^{\mathrm{calc}}=\frac{\sum_{i=1}^{N} 2 \pi \ell_{a i} \ell_{b i}}{\rho_{\mathrm{verm}}^{\mathrm{hydr}} \sum_{i=1}^{N} \pi h \ell_{a i} \ell_{b i}},
$$

where $\rho_{\text {verm }}^{\text {hydr }}$ is the mass density of hydrated vermiculite $\left(\rho_{\text {verm }}^{\text {hydr }}\right.$ $\left.=2.18 \mathrm{~g} \mathrm{~cm}^{3}\right)$. The lateral surface area $\left\langle A_{L}\right\rangle$ located at the curved edge of the same particles is given by

$$
\left\langle A_{L}\right\rangle^{\text {calc }}=\frac{\sum_{i=1}^{N} h \pi \sqrt{2\left(\ell_{a i}^{2}+\ell_{b i}^{2}\right)}}{\rho_{\text {verm }}^{\text {hydr }} \sum_{i=1}^{N} \pi h_{i} \ell_{a i} \ell_{b i}} .
$$




\section{$\underline{0.1-0.2 \text { size fraction }}$}

(a)

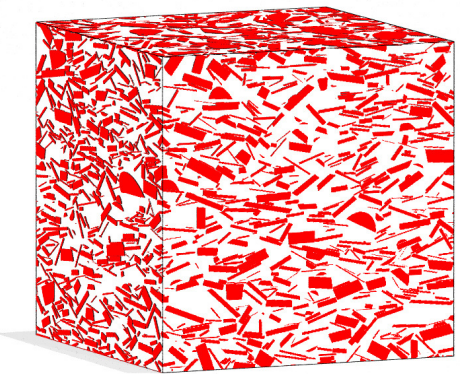

(b)

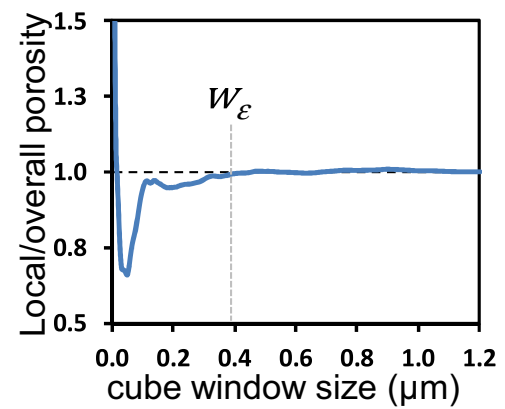

(c)

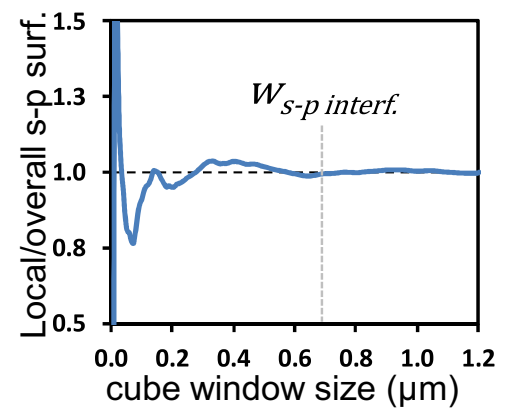

(c)
1-2 size fraction

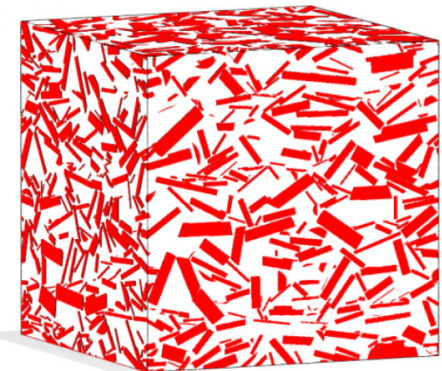

\section{0-20 size fraction}

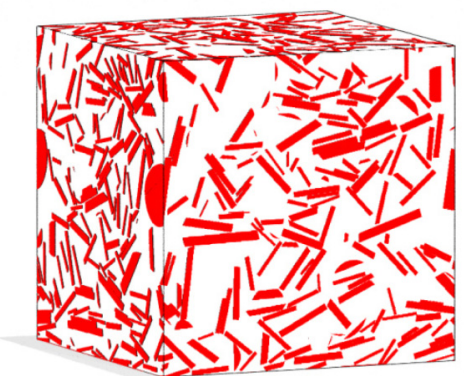

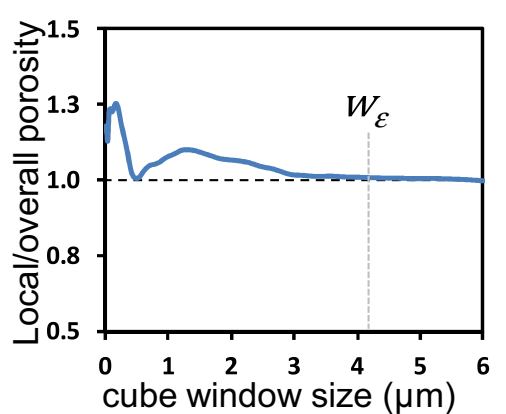

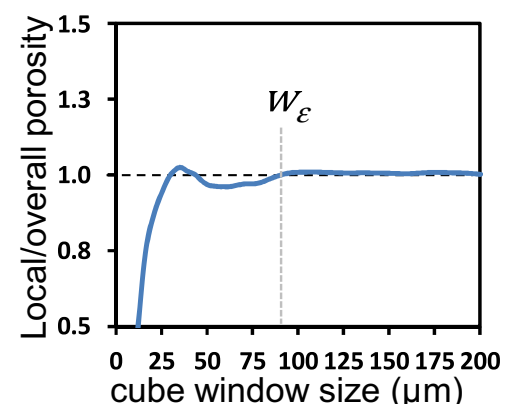

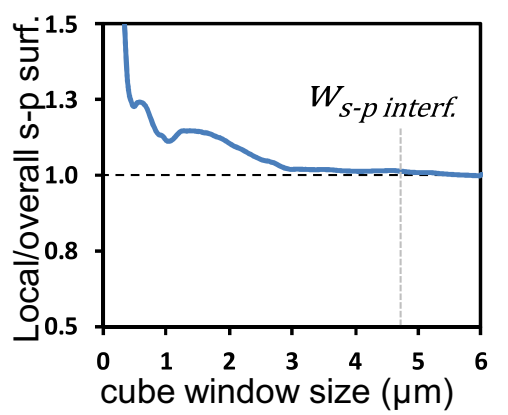

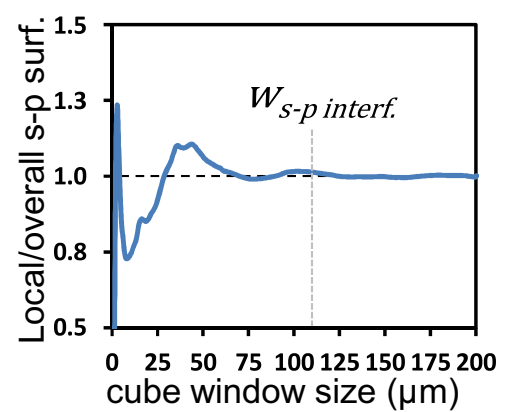

FIG. 11. (Color online) Properties of virtual-swelling clay porous media for the $0.1-0.2,1-2$, and 10-20 $\mu$ m particle size fractions (from left to right). (a) The $512^{3}$ resolution voxelized images of the virtual 3D packings shown in Fig. 9(a). (b) Representative elementary volume analysis of porosity parameter. (c) Same as (b) for the solid-pore interface area.

The total particle surface area is thus

$$
\left\langle A_{T}\right\rangle^{\text {calc }}=\left\langle A_{B}\right\rangle^{\text {calc }}+\left\langle A_{L}\right\rangle^{\text {calc }} .
$$

For all size fractions, the obtained values are successfully compared in Table IV with the experimental data derived from the study of Reinholdt et al. [34]. It should be emphasized, however, that the obtained $\left\langle A_{T}\right\rangle^{\text {calc }}$ values obtained from Eq. (10) take into account all the particle surfaces, even the parts that are in contact, and thus likely slightly overestimates the total pore-solid interface area. This latter parameter can be derived from $\left\langle A_{T}\right\rangle^{\text {calc }}$ values corrected based on the surface area lost due to contact between particles. Owing to the elliptic disk particle shape, these contact surfaces likely result from two particles in contact through their basal surfaces $\left\langle A_{B}\right\rangle_{\text {cont }}^{\text {calc }}$. The calculation of $\left\langle A_{B}\right\rangle_{\text {cont }}^{\text {calc }}$ for the different size fractions is achieved by first applying on the bottom basal surface of each particle a square grid of size $\delta_{m}$ and by calculating the minimum distance between the squares' centers and the basal surfaces of other particles. When this latter distance is less than the characteristic $d_{\text {cont }}$ value, the number of square centers $n_{s c}$ in contact is increased. At the end of the analysis for all particles with barycenter altitude $r_{z}$ found in the range $z_{\min }<r_{z} \leqslant z_{\max }$, the basal surface in contact is calculated as follows:

$$
\left\langle A_{B}\right\rangle_{\mathrm{cont}}^{\mathrm{calc}}=\frac{2 n_{s c} \delta_{m}^{2}}{\rho_{\mathrm{verm}}^{\mathrm{hydr}} \sum_{i=1}^{N} \pi h_{i} \ell_{a i} \ell_{b i}} .
$$

The obtained $\left\langle A_{B}\right\rangle_{\text {cont }}^{\text {calc }}$ values are reported in Table IV. It can be observed that the ratio $\left\langle A_{B}\right\rangle_{\text {cont }}^{\text {calc }} /\left\langle A_{B}\right\rangle^{\text {calc }}$ increases as the particle size decreases, a trend that is likely related to the more anisotropic organization for the finest particles, which favors surface contact between particles. However, the low $\left\langle A_{B}\right\rangle_{\text {cont }}^{\text {calc }}$ values obtained for all samples are consistent with the limited degree of anisotropy of the packings and their large $\varepsilon$ values, 
both effects having negative impact on the development of basal surface contact between particles. As a consequence, the total pore-solid interface areas, corresponding to $\left\langle A_{T}\right\rangle^{\text {calc }}$ values corrected from the contribution of $\left\langle A_{B}\right\rangle_{\text {cont }}^{\text {calc }}$ quantities, only differ from the original $\left\langle A_{B}\right\rangle^{\text {calc }}$ values by $\sim-6 \%$ for the three virtual porous media shown in Fig. 9(a).

The thorough comparison of the calculated and experimental data regarding the particles' surface and morphology [34] and particle orientation [32] indicates that the virtual 3D volume displayed in Fig. 9(a) is representative of the natural samples built from size-selected vermiculite fractions. It should be emphasized that other types of experimental data, such as pore-size distribution and porosity measurements, could have also been used for comparison. With respect to porosity, the obtained $\varepsilon$ values ranging from 0.63 to 0.71 are consistent with those obtained experimentally for mica [41,53], kaolinite [43], and montmorillonite [53] powders. However, caution should be taken in interpreting this agreement because the results reported in Fig. 6(b) indicate that for a given $S_{\text {part }}^{\text {calc }}$ value the porosity is strongly dependent on the morphology of the particles (see the discussion in Sec. III D).

\section{Representative elementary volume and chord length distribution analysis}

The representativeness of the obtained porous media allows for the investigation of specific properties of the systems that cannot be easily obtained experimentally, such as the anisotropic pore space geometry. Such analysis is valid only if the dimension $w$ chosen for the three size fractions is large enough to approach the representative elementary volume (REV), i.e., the minimum volume above which a given parameter of the porous medium becomes independent of sample size. The size of the REV strongly depends on the evaluated parameters, such as porosity, moisture saturation, and permeability [56-62]. Based on the $512^{3}$ resolution images reported in Fig. 11(a), a REV analysis is performed by analyzing the evolution of a given parameter of the porous medium within a core-centered cubic window whose size $\delta_{w}$ increases in all directions. In the present study, such an investigation is performed for both porosity and solid-pore surface areas. Although the voxelized 3D image can strongly affect the absolute surface value, it has only a limited effect on the REV analysis because the analysis focuses on the $\delta_{w}$ limit at which the surface value becomes invariant. The variations in the ratio between the local and overall porosity and the solid-pore surface as functions of $\delta_{w}$ are shown in Figs. 11(b) and 11(c), respectively, for the three samples. The REV size for porosity $w_{\varepsilon}$ and solid-pore surface $w_{s-p \text { interf }}$ is considered to be reached when the local porosity or solid-pore surface does not exceed $\pm 1 \%$ of the overall value. The $w_{\varepsilon}$ and

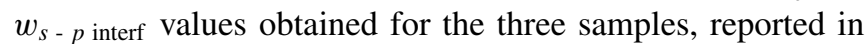
Table IV, are observed to be well contained in the simulation boxes. The porosity REV is smaller than the solid-pore surface parameter (Table IV), which agrees with numerous studies that have shown that the porosity parameter most often reaches the $\mathrm{REV}$ within a smaller volume than other properties of a porous medium [56-62].

Because the REV for the solid-pore interface area and porosity parameters is reached for all samples, chord length distribution analysis can be performed to provide a stereological description of the solid-pore interface [63-66]. The chord length distribution corresponds to the distribution of chord lengths connecting, for any direction $r$, two points on the pore-solid interface and lying entirely in the pore or solid phase. For the pore phase, the pore chord distribution $f_{p}(r)$ is defined such that $f_{p}(r) d r$ is the probability of finding a chord in the pore phase of a length between $r$ and $r+d r$ and by

$$
\int_{0}^{\infty} f_{p}(r) d r=1 .
$$

Based on both the pore chord distribution $f_{p}(r)$ and the solid chord distribution $f_{s}(r)$, one may calculate the mean chord length for both pore and solid phases along $r$ determined from the first momentum of the distribution function as follows:

$$
\begin{aligned}
& \ell_{p, \bar{r}}=\int_{0}^{\infty} r f_{p}(r) d r, \\
& \ell_{s, \bar{r}}=\int_{0}^{\infty} r f_{s}(r) d r,
\end{aligned}
$$

where $\ell_{p, \bar{r}}$ and $\ell_{s, \bar{r}}$ can be considered to reflect the mean sizes of pores and the solid phase along the same direction.

Calculations of pore and solid chord length distributions are performed using the 3D voxelized image reported in Fig. 11(a). The obtained $r f_{p}(r)$ and $r f_{s}(r)$ distributions plotted against $r$ in Figs. 12(a) and 12(b), respectively, are similar for the three size fractions along the $x$ and $y$ directions, in agreement with the uniaxial symmetry of the systems. In contrast, for a given size fraction, the shifts in both distributions towards lower $z$ values are consistent with the degree of anisotropy in particle orientation. Additional insights can be obtained from the $3 \mathrm{D}$ representation of mean chord length intercepts $\ell_{p, \bar{r}}$ and $\ell_{s, \bar{r}}$ reported in Fig. 12(c). For a given size fraction, the obtained mean chord length intercepts display a similar bell shape for the pore and solid phases, whereas the overall volumes between the representations of the two type of phases differ as a function of $\varepsilon$. This finding indicates that the pore space anisotropy is strongly related to the solid phase anisotropy. Such information can be quantified by calculating the ratios of mean chord length momentum between the $z$ and $x y$ directions as follows:

$$
\begin{aligned}
R_{p} & =\ell_{p, \bar{z}} / \ell_{p, \overline{x y}}, \\
R_{s} & =\ell_{s, \bar{z}} / \ell_{s, \overline{x y}} .
\end{aligned}
$$

The values of these ratios, reported in Table IV, further confirm that the pore and solid phases display similar anisotropic features. This degree of anisotropy can be compared to the orientation of particles in the porous media through the scalar order parameter $S_{\text {part }}^{\text {calc }}$ (cf. Table IV). For the 10-20 $\mu \mathrm{m}$ size fraction, the $R_{p}$ and $R_{s}$ values are close to 0.9 , indicating similar mean solid-pore surface interfaces along all directions, consistent with an $S_{\text {part }}^{\text {calc }}$ value close to 0.1 , which suggests a nearly isotropic particle orientation in the systems. Other size fractions follow this logical trend, in which both $R_{p}$ and $R_{s}$ decrease as $S_{\text {part }}^{\text {calc }}$ increases. Such a comparison allows for the analysis of the particle orientation parameter and stereological property of the pore space. The latter in particular is extremely difficult to access experimentally, especially for the finest 


\section{$\underline{0.1-0.2 \text { size fraction }}$}

(a)

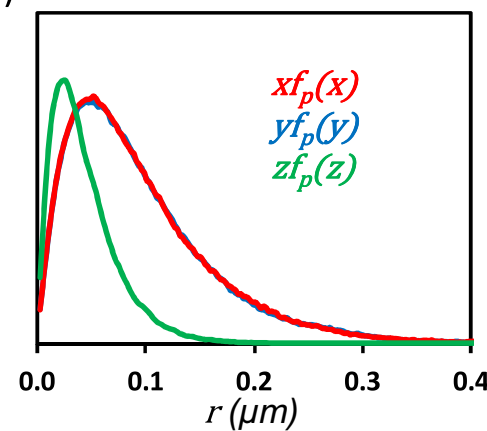

(b)

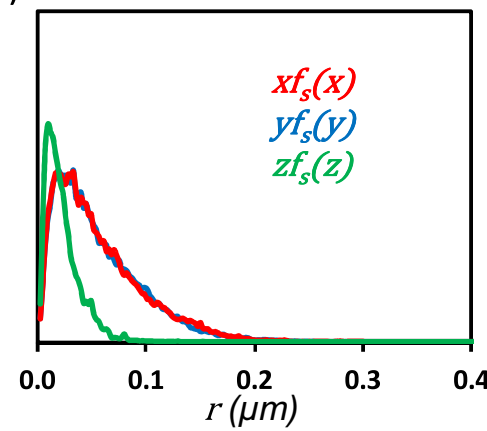

(c)

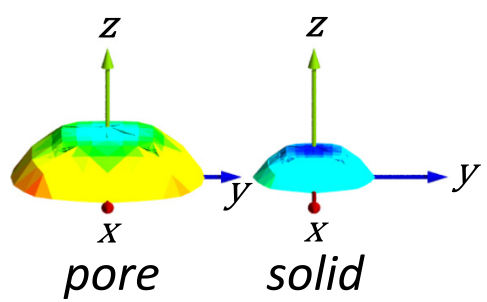

1-2 size fraction
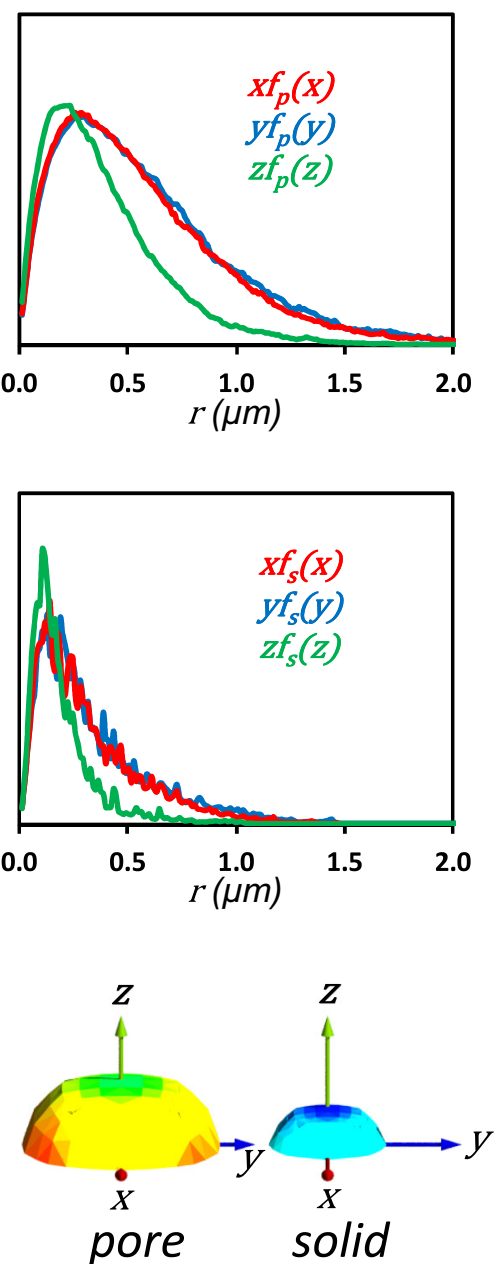

10-20 size fraction
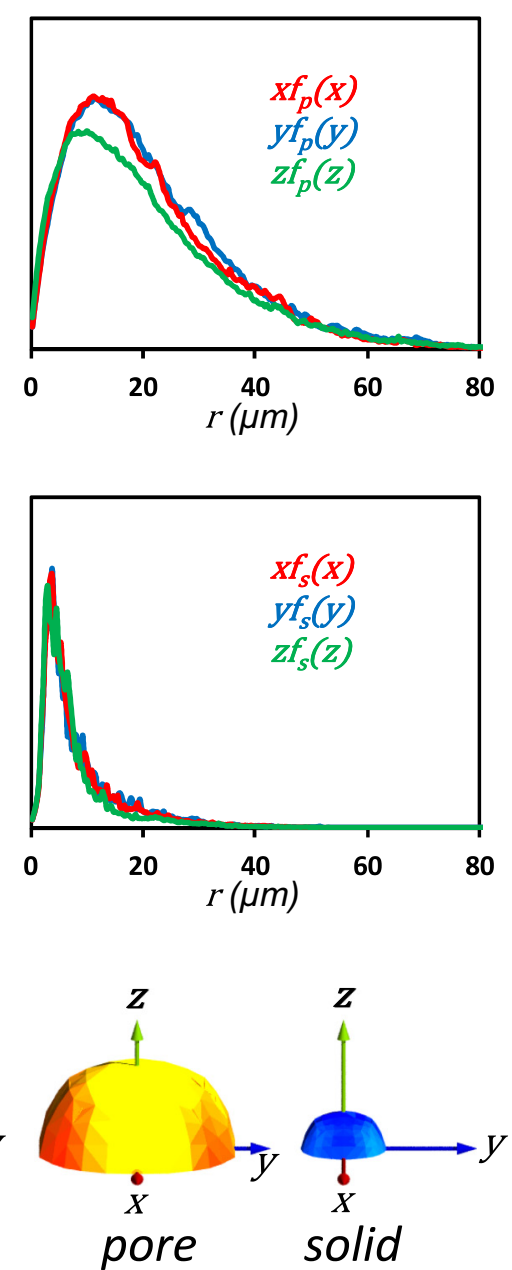

FIG. 12. (Color online) Properties of virtual-swelling clay porous media for the 0.1-0.2, 1-2, and 10-20 $\mu \mathrm{m}$ particle size fractions (from left to right). (a) Pore chord length analysis along the $x, y$, and $z$ directions, as shown from the obtained $r f_{p}(r)$ distributions. (b) Same analysis for the solid chord length distribution. (c) Three-dimensional representation of the mean chord length intercepts for the pore and solid phases $\left(\ell_{p, \bar{r}}\right.$ and $\ell_{s, \bar{r}}$, respectively).

particles, but represents a key parameter controlling the anisotropic features of water and solute diffusion commonly encountered in clay-based materials (see, e.g., [67-70]).

\section{CONCLUSION}

In the present study, a sequential deposition algorithm extending that developed by Coelho et al. [29] was applied to simulate geometrical properties of natural swelling-clay porous media. The results obtained for disks of different aspect ratios are consistent with published data. In addition, particular attention was devoted to a thorough analysis of particle orientation through the calculation of the scalar order parameter $S_{\text {part }}^{\text {calc }}$. Such an approach proves particularly relevant for flat disk packings because their porosities $\varepsilon$ fall onto a single master curve when plotted against $S_{\text {part }}^{\text {calc }}$. This result highlights the intimate relationship between $\varepsilon$ and $S_{\text {part }}^{\text {calc }}$ and demonstrates that, for strongly anisometric particles, both parameters must be considered jointly for an understanding of granular system geometrical properties. Furthermore, the $\varepsilon$ vs $S_{\text {part }}^{\text {calc }}$ relation likely represents the best way for comparing the ability of different algorithms to predict the lowest porosity in packings built from particles with a given aspect ratio.

Extension of the algorithm to particles with an elliptic disk morphology shows that the effect of the $\ell_{a} / \ell_{b}$ ratio is negligible for values less than 1.5. It is also observed that an increase in the width of the log-normal distribution of the aspect ratio and $\ell_{a} / \ell_{b}$ ratio leads to an increase in porosity. The reverse trend is obtained when the width of the particle size distribution is increased. Finally, application to natural swelling-clay porous media shows that the algorithm well reproduces different experimental data such as the distribution of particle morphology parameters (aspect ratio, size, and ratio $\ell_{a} / \ell_{b}$ ), macroscopic surface values, and the orientational properties of particles. Thus, the algorithms allows for the quantification of the different types of particle 
surfaces (lateral vs basal) accessible to solutes during diffusion processes in pore networks. In this regard, the sequential deposition algorithm could be used to shed light on the link between diffusional properties of water and solutes and various parameters of porous media, including those that can be easily determined by experiment, such as the order parameter $S^{\text {expt }}$.

\section{ACKNOWLEDGMENTS}

The Centre National de la Recherche Scientifique interdisciplinary défi Needs, through its MiPor program, is thanked for financial support provided to the present study (MPDYN project). The authors gratefully acknowledge the anonymous reviewers for their constructive comments.
[1] E. Ferrage, B. Lanson, L. J. Michot, and J. L. Robert, J. Phys. Chem. C 114, 4515 (2010).

[2] E. Ferrage, B. Sakharov, L. J. Michot, A. Delville, A. Bauer, B. Lanson, S. Grangeon, G. Frapper, M. Jiménez-Ruiz, and G. Cuello, J. Phys. Chem. C 115, 1867 (2011).

[3] B. Dazas, E. Ferrage, A. Delville, and B. Lanson, Am. Miner. 99, 1724 (2014).

[4] D. J. Cebula, R. K. Thomas, and J. W. White, Clays Clay Miner. 29, 241 (1981).

[5] J. J. Tuck, P. Hall, M. H. B. Hayes, D. K. Ross, C. Poinsignon, J. Chem. Soc. Faraday Trans. 80, 309 (1984).

[6] N. Malikova, A. Cadene, V. Marry, E. Dubois, P. Turq, J.-M. Zanotti, and S. Longeville, Chem. Phys. 317, 226 (2005).

[7] N. T. Skipper, P. A. Lock, J. O. Tililoye, J. Swenson, Z. A. Mirza, W. S. Howells, and F. Fernandez-Alonso, Chem. Geol. 230, 182 (2006).

[8] L. J. Michot, A. Delville, B. Humbert, M. Plazanet, and P. Levitz, J. Phys. Chem. C 111, 9818 (2007).

[9] H. N. Bordallo, L. P. Aldrifge, G. J. Churchman, W. P. Gates, M. T. F. Telling, N. Kiefer, P. Fouquet, T. Setdel, and S. A. J. Kimber, J. Phys. Chem. C 112, 13982 (2008).

[10] N. Malikova, E. Dubois, V. Marry, B. Rotenberg, and P. Turq, Z. Phys. Chem. 224, 153 (2010).

[11] F. G. Sanchez, T. Gimmi, F. Juranyi, L. van Loon, and L. Diamond, Environ. Sci. Technol. 43, 3487 (2009).

[12] V. Marry, E. Dubois, N. Malikova, S. Durand-Vidal, S. Longeville, and J. Breu, J. Environ. Sci. Technol. 45, 2850 (2011).

[13] W. P. Gates, H. N. Bordallo, L. P. Aldridge, T. Seydel, H. Jacobsen, V. Marry, and G. J. J. Churchman, J. Phys. Chem. C 116, 5558 (2012).

[14] M. Jiménez-Ruiz, E. Ferrage, A. Delville, and L. J. Michot, J. Phys. Chem. A 116, 2379 (2012).

[15] L. J. Michot, E. Ferrage, M. Jiménez-Ruiz, M. Boehm, and A. Delville, J. Phys. Chem. C 116, 16619 (2012).

[16] V. Marry, E. Dubois, N. Malikova, J. Breu, and W. Haussler, J. Phys. Chem. C 117, 15106 (2013).

[17] S. Mitra, S. A. Prabhudesai, D. Chakrabarty, V. K. Sharma, M. A. Vicente, J. P. Embs, and R. Mukhopadhyay, Phys. Rev. E 87, 062317 (2013).

[18] M. L. Martins, W. P. Gates, L. J. Michot, E. Ferrage, V. Marry, and H. N. Bordallo, Appl. Clay Sci. 96, 22 (2014).

[19] K. D. Knudsen, J. O. Fossum, G. Helgesen, and M. W. Haakestad, Physica B 352, 247 (2004).

[20] Y. Méheust, K. D. Knudsen, and J. O. Fossum, J. Appl. Crystallogr. 39, 661 (2006).

[21] Y. Méheust, S. Dagois-Bohy, K. D. Knudsen, and J. O. Fossum, J. Appl. Crystallogr. 40, s286 (2007).

[22] G. Vasseur, I. Djeranmaigre, D. Grunberger, G. Rousset, D. Tessier, and B. Velde, Mar. Petrol. Geol. 12, 941 (1995).
[23] S. V. Churakov and T. Gimmi, J. Phys. Chem. C 115, 6703 (2011).

[24] S. V. Churakov, T. Gimmi, T. Unruh, L. Van Loon, and F. Juranyi, Appl. Clay Sci. 96, 36 (2014).

[25] L. Holzer, B. Münch, M. Rizzi, R. Wepf, P. Marschall, and T. Graule, Appl. Clay Sci. 47, 330 (2010).

[26] S. Tomioka, T. Kozaki, H. Takamatsu, N. Noda, S. Nisiyama, N. Kozai, S. Suzuki, and S. Sato, Appl. Clay Sci. 47, 65 (2010).

[27] L. Holzer and M. Cantoni, in Nanofabrication Using Focused Ion and Electron Beams: Principles and Applications, edited by I. Utke, S. A. Moshkalev, and P. Russel (Oxford University Press, New York, 2012).

[28] L. M. Keller, A. Seiphoori, P. Gasser, F. Lucas, L. Holzer, and A. Ferrari, Clays Clay Miner. 62, 174 (2014).

[29] D. Coelho, J.-F. Thovert, and P. M. Adler, Phys. Rev. E 55, 1959 (1997).

[30] I. Malinouskaya, V. V. Mourzenko, J.-F. Thovert, and P. M. Adler, Phys. Rev. E 80, 011304 (2009).

[31] V. Mourzenko, J.-F. Thovert, O. Vizika, and P. M. Adler, Phys. Rev. E 77, 066306 (2008).

[32] F. Hubert, I. Bihannic, D. Prêt, E. Tertre, B. Nauleau, M. Pelletier, B. Demé, and E. Ferrage, Clays Clay Miner. 61, 397 (2013).

[33] F. Hubert, L. Caner, A. Meunier, and E. Ferrage, Am. Miner. 97, 384 (2012).

[34] M. X. Reinholdt, F. Hubert, M. Faurel, E. Tertre, A. Razafitianamaharavo, G. Francius, D. Prêt, S. Petit, E. Béré, M. Pelletier, and E. Ferrage, Appl. Clay Sci. 77-78, 18 (2013).

[35] L. Rothenburg and R. J. Bathurst, Comput. Geotechnol. 11, 315 (1991).

[36] G. W. Delaney, D. Weaire, S. Hutzler, and S. Murphy, Philos. Mag. Lett. 85, 89 (2005).

[37] Version 3.7, retrieved from http://www.povray.org.

[38] D. Coelho, Ph.D. thesis, Poitiers, 1996 (unpublished).

[39] P. W. Cleary and M. L. Sawley, Appl. Math. Model. 26, 89 (2002).

[40] F. Y. Fraige, P. A. Langston, and G. Z. Chen, Powder Technol. 186, 224 (2008).

[41] J.-H. Kim, J. A. Ochoa, and S. Whitaker, Transport Porous Med. 2, 327 (1987).

[42] R. P. Zou and A. B. Yu, Powder Technol. 88, 71 (1996).

[43] A. C. Perdigon-Aller, M. Aston, and S. M. Clarke, J. Colloid Interface Sci. 290, 155 (2005).

[44] X. Jia and R. A. Williams, Powder Technol. 120, 175 (2001).

[45] X. Jia, M. Gan, R. A. Williams, and D. Rhodes, Powder Technol. 174, 10 (2007).

[46] G. W. Delaney, J. E. Hilton, and P. W. Cleary, Phys. Rev. E 83, 051305 (2011). 
[47] F. M. Schaller, M. Neudecker, M. Saadatfar, G. W. Delaney, G. E. Schröder-Turk, and M. Schröter, Phys. Rev. Lett. 114, 158001 (2015).

[48] A. Donev, I. Cisse, D. Sachs, E. A. Variano, F. H. Stillinger, R. Connelly, S. Torquato, and P. M. Chaikin, Science 303, 990 (2004).

[49] A. Donev, F. H. Stillinger, and S. Torquato, J. Comput. Phys. 202, 737 (2005).

[50] A. Donev, S. Torquato, F. H. Stillinger, and R. Connelly, J. Appl. Phys. 95, 989 (2004).

[51] M. Chaikin and T. C. Lubensky, Principles of Condensed Matter Physics (Cambridge University Press, Cambridge, 2000).

[52] R. Eppenga and D. Frenkel, Mol. Phys. 52, 1303 (1984).

[53] N. Mammar, M. Rosanne, B. Prunet-Foch, J.-F. Thovert, E. Tevissen, and P. M. Adler, J. Colloid Interface Sci. 240, 498 (2001).

[54] D. J. Cebula, R. K. Thomas, S. Middleton, R. H. Ottewill, and J. W. White, Clays Clay Miner. 27, 39 (1979).

[55] P. L. Hall, R. Harrison, M. H. B. Hayes, J. J. Tuck, and D. K. Ross, J. Chem. Soc. Faraday Trans. 179, 1687 (1983).

[56] P. Baveye and G. Sposito, Water Resour. Res. 20, 521 (1984).

[57] J. Bear and Y. Bachmat, Introduction to Modeling of Transport Phenomena in Porous Media (Kluwer Academic, Dordrecht, 1990).
[58] D. Zhang, R. Zhang, S. Chen, and W. E. Soll, Geophys. Res. Lett. 27, 1195 (2000).

[59] M. Stroeven, H. Askes, and L. J. Sluys, Comput. Methods Appl. Mech. Eng. 193, 3221 (2004).

[60] A. Salama and P. J. Van Geel, J. Porous Media 11, 403 (2008).

[61] R. Al-Raoush and A. Papadopoulos, Powder Technol. 200, 69 (2010).

[62] M. S. Costanza-Robinson, B. D. Estabrook, and D. F. Fouhey, Water Resour. Res. 47, W07513 (2011).

[63] P. Levitz and D. Tchoubar, J. Phys. I France 2, 771 (1992).

[64] S. Torquato and B. Lu, Phys. Rev. E 47, 2950 (1993).

[65] I. Cousin, P. Levitz, and A. Bruand, Eur. J. Soil Sci. 47, 439 (1996).

[66] O. Rozenbaum, Sci. Total Environ. 409, 1959 (2011).

[67] M. Rosanne, N. Mammar, N. Koudina, B. Prunet-Foch, J.-F. Thovert, E. Tevissen, and P. M. Adler, J. Colloid Interface Sci. 260, 195 (2003).

[68] L. R. Van Loon, J. M. Soler, W. Müller, and M. H. Bradbury, Environ. Sci. Technol. 38, 5721 (2004).

[69] H.-R. Wenk, M. Voltolini, M. Mazurek, L. R. Van Loon, and A. Vinsot, Clays Clay Miner. 56, 285 (2008).

[70] J.-C. Robinet, P. Sardini, D. Coelho, J.-C. Parneix, D. Prêt, S. Sammartino, E. Boller, and S. Altmann, Water Resour. Res. 48, W05554 (2012). 